\title{
Impact of The Correlation Between Channel Input And Side Information On Physical Layer Security Performances of Wireless Wiretap Channel
}

\author{
Saeid Pakravan ( $\nabla$ saeidpakravan1991@gmail.com ) \\ Ferdowsi University of Mashhad \\ Ghosheh Abed Hodtani \\ Ferdowsi University of Mashhad https://orcid.org/0000-0002-4337-1624
}

\section{Research Article}

Keywords: Fading wiretap channel, Secrecy capacity, Secrecy outage probability, Correlated side information

Posted Date: June 28th, 2021

DOI: https://doi.org/10.21203/rs.3.rs-504684/v1

License: (c) (i) This work is licensed under a Creative Commons Attribution 4.0 International License. Read Full License 


\title{
Impact of the Correlation Between Channel Input and Side Information on Physical Layer Security Performances of Wireless Wiretap Channel
}

\author{
Saeid Pakravan, Ghosheh Abed Hodtani \\ Department of Electrical Engineering, Ferdowsi University of Mashhad, Mashhad, Iran \\ Email: Saeid.pakravan@mail.um.ac.ir, hodtani@um.ac.ir
}

\begin{abstract}
In this paper, a discrete memoryless wiretap channel with non-causal side information known at the encoder is considered. We (i) characterize capacity region for the Gaussian version of this channel by considering correlation between channel input and side information available at the transmitter; (ii) analyze the impact of correlation on the performance of physical layer security in a Rayleigh fading wiretap channel by deriving closed-form expressions on the average secrecy capacity (ASC) and secrecy outage probability (SOP). Further, to more show the impact of side information, asymptotic behavior of SOP is studied. Numerical evaluation of theoretical results is done finally.
\end{abstract}

Keywords- Fading wiretap channel, Secrecy capacity, Secrecy outage probability, Correlated side information.

\section{INTRODUCTION}

Due to the increasing use of wireless communication systems, there are many challenges on the security situations of these networks. In communication systems, security usually has been considered as the upper layer and utilized through implementing cryptographic algorithms, assuming that computational capabilities are constrained [1]. However, this assumption may not be accurate due to the expansion in computational methods resulting in breaking encryption algorithms. Therefore, physical layer security is known as theoretical information security to protect information against unauthorized access and also to ensure secure communication. The issue of physical layer security was first reported by Shannon in [2]. Shannon studied the secure communication and demonstrated that it can be achieved by a secret key shared between the transmitter and the receiver when the entropy of key is greater than or equal to the entropy of the message to be transmitted. In [3], Wyner studied the degraded wiretap channel (WC) and proved that secure transmission is achievable in the absence of a secret key; the WC is a channel where a transmitter forwards a message to a receiver while the message is kept secret from an eavesdropper. In this work, the maximum information rate of the main channel (transmitter-to-legitimate receiver) with the total ignorance at the eavesdropper has been defined as a secrecy capacity (SC) and it has been demonstrated the positive information rate can be achieved under the assumption that the eavesdropper channel (transmitter to- eavesdropper) is a degraded version of the main channel. Later, Csiszar and Korner in [4] extended Wyner's result to the general BC (not necessarily degraded) with confidential messages and established the SC. The authors in [5] generalized the results of [3] to the Gaussian version and defined the SC as the difference between capacities of the main and eavesdropper channels. Other researchers have been studied the importance of secrecy efficiency metrics over various fading channels [6$16]$.

The side information (SI) is a general concept studied in the various communication scenarios. Shannon studied channels with SI and found the capacity of the single-user channel when causal SI is available at the transmitter [17]. Single user channel, when non-causal SI is available only at the transmitter, was studied by Gel'fand and Pinsker in [18]. The results of [17] has been generalized to the case where non-causal SI is available at both transmitter and receiver in [19]. The Gaussian versions of [18] were studied in [20] and [21]. Recently, this concept was demonstrated for the WC and several works have been done upon this set of channels [22-25]. In [22] an achievable rate equivocation region for the discrete memoryless WC with respect to SI is presented and also the following results are generalized to the Gaussian case. In [23] a lower bound on the SC of the WC with considering SI available causally at both the encoder and decoder has been established. In [24] an achievable rate equivocation region for a WC (not necessarily degraded) with two-sided channel SI available at the transmitter and the legitimate receiver has been established. Authors in [25] have illustrated how SI and interference affect the secret communications.

Our work. The information-theoretic study of the impact of the cognition on the channel capacity in the multiuser channel is an important and interesting idea. In some communications scenarios, channel input and SI may be correlated and therefore, they can be modeled as correlated random variables (RVs). In this paper we will analyze the effect of the correlation between the SI known at the transmitter and the input of the channel. There are practically and theoretically important situations that this assumption is true. One example is physical layer security for a measurement system where the measuring signal may 
affect the system under measurement. Some communication scenarios in which the channel input and the SI may be correlated and the related investigations can be found in [2932]. Here, we study the impact of correlation between the channel input and the SI on the ASC, and SOP of the Rayleigh fading WC by expressions in terms of series and eventually compare the achieved results with the case without dependency and also without SI. Afterwards, we analyze the asymptotic behavior of SOP for high value of the average SNRs.

The remaining parts of this paper are formed as follows. The system model and definitions are discussed in Section II. In Section III, SC for the wireless WC with considering SI available at the transmitter and correlation between the channel input and the SI is provided, and then, the ASC and SOP of this case are investigated. Numerical results for illustrating the impact of correlation between the channel input and the SI on the physical layer security performances are studied in Section VI. Finally, we conclude the paper in Section V.

Notations: we use upper case letters for RVs, lower case letters for the realization of RVs, and calligraphic letters denote the alphabet set. Max $\min \{\cdot\}$ represents maximizing the minimization of a function. Mutual information between random variables $X$ and $Y$ is denoted by $I(X ; Y)$.

\section{ChanNel Model AND Definitions}

The channel model and some basic necessary definitions that we are going to work upon have been characterized in this section. A wireless channel is studied here, where a transmitter needs to send a confidential message to a legitimate receiver while the eavesdropper is trying to decode the message from its received signal by knowing the SI known in the transmitter as depicted in Fig. 1.

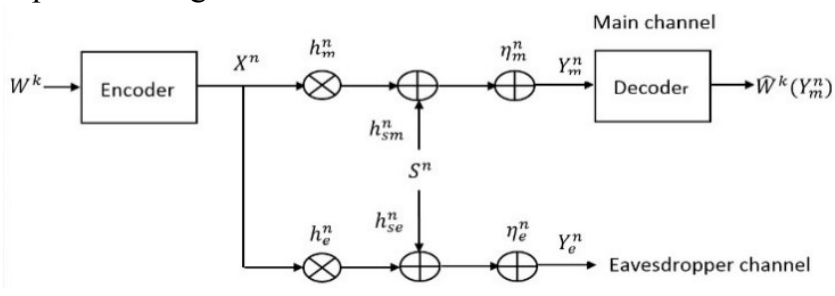

Fig. 1. An uncorrelated Rayleigh fading WC.

Let $\mathcal{X}$ be the input set, $\mathcal{Y}_{m}$ be the output set of legitimate receiver, $\mathcal{Y}_{e}$ be the output set of eavesdropper and $\mathcal{S}$ be finite set that denotes SI in the transmitter. The SI is non-causally known at the encoder and $S_{i}, 1 \leq i \leq n$, are independently and identically distributed (i.i.d. $) \sim p(s)$. We want to forward a message $W^{k} \in\{1,2, \ldots, M\}$ to the legitimate receiver in $n$ uses of the channel. Due to the $W^{k}$ and $S^{n}$, the encoder forwards a codeword $X^{n}$ to the main channel. Upon obtaining of $Y_{m}^{n}$ the decoder at the legitimate receiver creates an estimate $\widehat{W}^{k}\left(Y_{m}^{n}\right)$ of the message $W^{k}$. The corresponding output at the eavesdropper is $Y_{e}^{n}$. So, the received signals in Rayleigh fading WC by considering legitimate receiver as $Y_{m}(i)$ and eavesdropper as $Y_{e}(i)$ can be specified as follows:

$$
Y_{m}(i)=h_{m}(i) X(i)+h_{s m}(i) S(i)+\eta_{m}(i)
$$

$$
Y_{e}(i)=h_{e}(i) X(i)+h_{s e}(i) S(i)+\eta_{e}(i), i=1, \ldots, n
$$

where $n$ is the length of the transmitted signal, $\eta_{m}(i)$ and $\eta_{e}(i)$ are i. i.d. additive white Gaussian noise (AWGN) with zero mean and variances $N_{m}$ and $N_{e}$ respectively. The channels between the transmitter to legitimate receiver, and transmitter to eavesdropper have fading coefficients, indicated by $h_{m}$ and $h_{e}$, respectively. The information $\mathrm{S}$ is received by legitimate receiver and eavesdropper through $h_{s m}$ and $h_{s e}$ coefficients respectively, which are necessarily not equal to $h_{m}$ or $h_{e}$. To consider a Rayleigh fading scenario, all received signals are assumed to be attenuated by a Rayleigh fading channel coefficients which are constant during a transmission block (block fading) and they are known in all transmitter and receivers. So they are fixed in each block (i.e., $h_{m}(i)=h_{m}$, $h_{s m}(i)=h_{s m}, h_{s e}(i)=h_{s e}$ and $\left.h_{e}(i)=h_{e}, \forall i=1, \ldots, n\right)$, and vary randomly from one block to another block. This concept means that the channel power gains (i.e., $g_{m}(i)=$ $\left|h_{m}(i)\right|^{2}, g_{s m}(i)=\left|h_{s m}(i)\right|^{2}, g_{s e}(i)=\left|h_{s e}(i)\right|^{2}$ and $g_{e}(i)=$ $\left.\left|h_{e}(i)\right|^{2}\right)$ are distributed exponentially. Moreover, it has been supposed that the codewords forward by transmitter over the channels are constrained to the average power limitation as:

$$
\frac{1}{n} \sum_{i=1}^{n} \mathrm{E}\left\{\left|X_{i}\right|^{2}\right\} \leq P,
$$

where the average power of the transmission signal is displayed with $P$. The instantaneous SNR at legitimate receiver and eavesdropper when there is not any SI at the transmitter are respectively specified as $\gamma_{m}(i)=\frac{P\left|h_{m}(i)\right|^{2}}{N_{m}}=\frac{P\left|h_{m}\right|^{2}}{N_{m}}=\gamma_{m}$ and $\gamma_{e}(i)=\frac{P\left|h_{e}(i)\right|^{2}}{N_{e}}=\frac{P\left|h_{e}\right|^{2}}{N_{e}}=\gamma_{e} ;$ and the average SNR at legitimate receiver and eavesdropper are respectively given as $\overline{\gamma_{m}}(i)=\frac{P E\left[\left|h_{m}(i)\right|^{2}\right]}{N_{m}}=\frac{P E\left[\left|h_{m}\right|^{2}\right]}{N_{m}}=\overline{\gamma_{m}}$ and $\overline{\gamma_{e}}(i)=\frac{P E\left[\left|h_{e}(i)\right|^{2}\right]}{N_{e}}=$ $\frac{P E\left[\left|h_{e}\right|^{2}\right]}{N_{e}}=\overline{\gamma_{e}}$. Since in the quasi-static case $h_{m}$ and $h_{e}$ are random but remain constant for all time, it is entirely appropriate to see the main channel and the eavesdropper's channel as a complex AWGN channel with SNRs $\gamma_{m}=\frac{P\left|h_{m}\right|^{2}}{N_{m}}$ and $\gamma_{e}=\frac{P\left|h_{e}\right|^{2}}{N_{e}}$ respectively. It is as well as advantageous to bring up the probability density function of $\gamma_{m}$ and $\gamma_{e}$. Since the channel fading coefficients $h$ are zero-mean complex Gaussian RVs and the instantaneous SNR $\gamma \propto|h|^{2}$, it follows that $\gamma$ is distributed exponentially, in particular

and

$$
f\left(\gamma_{m}\right)=\frac{1}{\overline{\gamma_{m}}} \exp \left(-\frac{\gamma_{m}}{\overline{\gamma_{m}}}\right),
$$

$$
f\left(\gamma_{e}\right)=\frac{1}{\overline{\gamma_{e}}} \exp \left(-\frac{\gamma_{e}}{\overline{\gamma_{e}}}\right) .
$$

In considered system model, the transmission rate between transmitter and legitimate receiver is indicated as $R=\frac{H(M)}{n}$. The equivocation rate of eavesdropper that illustrates the secrecy level of confidential messages versus eavesdropper is 
characterized as $R_{e}=\frac{1}{n} H\left(W \mid Z^{n}\right)$. Also, we define average probability of error as follows.

$$
P_{e}^{a v g} \triangleq \frac{1}{M} \sum_{i=1}^{n} \operatorname{Pr}\left(\widehat{W}^{k}\left(Y_{m}^{n}\right) \neq i \mid W^{k}=i\right),
$$

where $\widehat{W}^{k}\left(Y_{m}^{n}\right)$ denotes estimated messages by legitimate receiver. The secrecy rate $R_{S}$ is defined to be achievable, if there exists a code $\left(2^{n R_{S}}, n\right)$ so that for all $\epsilon \geq 0$ and sufficiently large $n, P_{e}^{a v g} \leq \epsilon$ and $R_{e} \geq R_{s}-\epsilon$, where, $R_{e}$ and $P_{e}^{a v g}$ have been defined before this. The SC $C_{S}$ can be defined as

$$
C_{s}=\sup _{P_{e}^{a v g}} R_{s} R_{s}
$$

In our paper, we assume that SI exists at the transmitter and define $\quad \gamma_{s m}(i)=\frac{Q\left|h_{s m}(i)\right|^{2}}{N_{m}}=\frac{Q\left|h_{s m}\right|^{2}}{N_{m}}=\gamma_{s m}, \quad \overline{\gamma_{s m}}(i)=$ $\frac{Q E\left[\left|h_{s m}(i)\right|^{2}\right]}{N_{m}}=\frac{Q E\left[\left|h_{s m}\right|^{2}\right]}{N_{m}}=\overline{\gamma_{s m}}, \gamma_{s e}(i)=\frac{Q\left|h_{s e}(i)\right|^{2}}{N_{e}}=\frac{Q\left|h_{s e}\right|^{2}}{N_{e}}=$ $\gamma_{s e}$, and $\overline{\gamma_{s e}}(i)=\frac{Q E\left[\left|h_{s e}(i)\right|^{2}\right]}{N_{e}}=\frac{Q E\left[\left|h_{s e}\right|^{2}\right]}{N_{e}}=\overline{\gamma_{s e}}$. We have following marginal distributions for $\gamma_{s m}>0$ and $\gamma_{s e}>0$, respectively as

and

$$
f\left(\gamma_{s m}\right)=\frac{1}{\overline{\gamma_{s m}}} \exp \left(-\frac{\gamma_{s m}}{\overline{\gamma_{s m}}}\right),
$$

$$
f\left(\gamma_{s e}\right)=\frac{1}{\overline{\gamma_{s e}}} \exp \left(-\frac{\gamma_{s e}}{\overline{\gamma_{s e}}}\right)
$$

The effectual meaning of this set of definitions and as well as the significations of the basic assumptions will be explained in the next section. Note that the Gaussian WC with correlation between the channel input and SI is an expansion of the dirty paper channel by accompanying by an eavesdropper. Using an analogous approach of writing on dirty paper, we will have the following communication problem: the transmitter wants to forward a secret message to a receiver and he knows existence of an eavesdropper. He writes the secret message on a paper using a limited amount of ink and forwards it. There are a correlation between the channel input and the SI. Along the way to the legitimate receiver, the paper obtains the desired distribution dirt; also, it is assumed that eavesdropper has access to the paper and its distribution. Now the question of our interest is: how much is dependency between channel input and SI effect on the physical layer security performances for the wireless WC?

\section{MAIN RESULTS}

In this section, firstly, for the channel defined in the previous section, related works on SC are presented. Then, (i) an achievable secrecy rate region and an outer bound on the SC region are obtained, and, (ii) the discrete alphabet results are extended to the continuous alphabet wireless WC by considering correlation between the channel input and SI; Finally, (iii) the impact of this dependency over ASC and SOP are analyzed.

\section{A. Related works}

The discrete memoryless lower bound on SC for the WC with considering SI at the transmitter has been provided in [22], as follows:
$R_{S}^{\text {low }}=\max _{U \rightarrow(X, S) \rightarrow Y_{m} \rightarrow Y_{e}} I\left(U ; Y_{m}\right)-\max \left\{I(U ; S), I\left(U ; Y_{e}\right)\right\}$,

where $U$ is auxiliary $\mathrm{RV}$ such that $U \rightarrow(X, S) \rightarrow Y_{m} \rightarrow Y_{e}$ forms a Markov chain. An upper bound on the SC of the WC with considering SI at the transmitter has been provided in [22], as follows:

$$
R_{s}^{u p}=\min \left\{C_{m}^{\prime} \max _{U \rightarrow(X, S) \rightarrow Y_{m} \rightarrow Y_{e}}\left[I\left(U ; Y_{m}\right)-I\left(U ; Y_{e}\right)\right]\right\}
$$

where $C_{m}$ is the capacity of the main channel with considering SI and it is defined as:

$$
C_{m}=\max _{U \rightarrow(X, S) \rightarrow Y_{m} \rightarrow Y_{e}}\left[I\left(U ; Y_{m}\right)-I(U ; S)\right] .
$$

Corollary 1 . If there exists an auxiliary parameter $U_{m}$ such that

1. $U_{m} \rightarrow(X, S) \rightarrow Y_{m} \rightarrow Y_{e}$ forms a Markov chain,

2. $I\left(U_{m} ; Y_{m}\right)-I\left(U_{m} ; S\right)=C_{m}$,

3. $I\left(U_{m} ; S\right) \geq I\left(U_{m} ; Y_{e}\right)$,

then, for the discrete memoryless WC with SI, the secrecy capacity $C_{s}$ is equal to $C_{m}$.

Corollary 2. If there exists an auxiliary parameter $U_{e}$ such that

1. $U_{e} \rightarrow(X, S) \rightarrow Y_{m} \rightarrow Y_{e}$ forms a Markov chain,

2. $I\left(U_{e} ; Y_{m}\right)-I\left(U_{e} ; Y_{e}\right)=R_{e_{S}}$,

3. $I\left(U_{e} ; Y_{e}\right) \geq I\left(U_{e} ; S\right)$,

then, for the discrete memoryless WC with SI, the secrecy capacity $C_{s}$ is equal to $R_{e_{s}}$.

Lemma 1. The conditions for the above corollaries 1 and 2 are explicitly determined based on the system model parameters as

$$
\begin{gathered}
I(U ; S) \geq I\left(U ; Y_{e}\right) \leftrightarrow \alpha \geq \alpha_{0} \text { or } \alpha \leq \alpha_{-0}, \\
\left(U ; Y_{e}\right) \geq I(U ; S) \leftrightarrow \alpha_{-0} \leq \alpha \leq \alpha_{0},
\end{gathered}
$$

where $\alpha_{0}=\left(1-\frac{N_{e}}{P+N_{e}}\right)\left(1+\sqrt{1+\frac{P+N_{e}}{Q}}\right)$, and $\alpha_{-0}=(1-$ $\left.\frac{N_{e}}{P+N_{e}}\right)\left(1-\sqrt{1+\frac{P+N_{e}}{Q}}\right)$.

Proof. The details of proof are in [22].

Now, suppose random variable $(X, S)$ are jointly Gaussian distributed. The channel input $X$ is correlated to $S$ with coefficient $\rho_{X S}=\frac{E(X, S)}{\sqrt{P Q}}$ and $0 \leq \rho_{X S} \leq 1$. We consider complex fading coefficients for both the main channel and the eavesdropper's channel, as detailed in Section II.

\section{B. Our Works}

In this section, first, in subsection B1, we obtain SC for wireless $\mathrm{WC}$, and then, in subsection B2, secrecy concepts of ASC and SOP are analyzed.

\section{B1. Secrecy capacity of the wireless wiretap channel}

In this sub-section, we derive $\mathrm{SC}$ of the wireless $\mathrm{WC}$ with considering SI at the transmitter. According to the system model illustrated in section II and results in corollaries 1 and 2 the following theorems provide SC for the wireless WC with SI.

Theorem 1: The SC $C_{S}$ of the wireless WC with SI at the transmitter, and fading coefficients satisfying the above 
Corollaries 1 and 2 in section $A$, and existing correlation between channel input and SI is given as follows.

$C_{S}$

$$
=\left\{\begin{array}{cc}
\log \left(1+\left(1-\rho_{X S}^{2}\right) \gamma_{m}\right), & \text { if Corollary 1 } \\
{\left[\log \left(\frac{1+\gamma_{m}+\gamma_{s m}+2 \rho_{X s} \sqrt{\gamma_{m} \gamma_{s m}}}{1+\gamma_{e}+\gamma_{s e}+2 \rho_{X s} \sqrt{\gamma_{e} \gamma_{s e}}}\right)\right]^{+},} & \text {if Corollary 2 }
\end{array}\right.
$$

where $[x]^{+}=\max (0, x)$.

Proof: The details of proof are in Appendix A.

\section{B2. ASC and SOP analysis}

In this sub-section, we derive, through Theorem 1, the ASC and SOP for the uncorrelated Rayleigh fading WCs with SI available at the transmitter and is correlated to channel input $X$ with coefficient $\rho_{X S}$.

ASC analysis. In our considered channel model, the main channel and eavesdropper channel are assumed block fading channels with complex noise and complex fading coefficients. So, regards to the results obtained in previous sub-section, the $\mathrm{SC}$ for a complex AWGN-WC is defined as Theorem 1. Thus, for a wireless WC with random SNRs, when the eavesdropper channel is noisier than the main channel (i.e. $N_{e}>N_{m}$ which also gives results $\gamma_{s e}<\gamma_{s m}$ ) and the SNR at eavesdropper is lower than the SNR at legitimate receiver (i.e., $\gamma_{e}<\gamma_{m}$ ), we can derive the ASC or $C_{s}^{a v g}$ for Corollary 1 and 2 as the following theorems. The fading channels are assumed to be uncorrelated.

Theorem 2. The ASC for concerned uncorrelated Rayleigh fading WC with defined parameters $\overline{\gamma_{m}}, \overline{\gamma_{e}}, \overline{\gamma_{s m}}$ and $\overline{\gamma_{s e}}$ that the channel input $X$ is correlated to $S$ with coefficient $\rho_{X S}$, is given as

For Corollary 1:

For Corollary 2:

$$
C_{s}^{a v g}=\frac{\sqrt{\pi}}{2} \exp \left(\frac{1-\frac{16}{\pi^{2}}}{\left(1-\rho_{X S}^{2}\right) \overline{\gamma_{m}}}\right)
$$

$C_{s}^{a v g}=\frac{\sqrt{\pi}}{2\left(1-\overline{\gamma_{m} \gamma_{s m}}\right)} e^{\frac{1-\frac{16}{\pi^{2}}}{\overline{\gamma_{m}}}}-\frac{\sqrt{\pi}}{2}\left(\frac{1}{1+\frac{\overline{\gamma_{m}}}{\overline{\gamma_{e}}}-\frac{\overline{\gamma_{m}}}{\overline{\gamma_{s m}}}}-\frac{1}{1-\frac{\overline{\gamma_{m}}}{\overline{\gamma_{s m}}}}\right)$ $. e^{\frac{1-\frac{16}{\pi^{2}}}{\bar{\gamma}_{s m}}}-\frac{\sqrt{\pi} \overline{\gamma_{s e}}}{2\left(\overline{\gamma_{s e}}-\overline{\gamma_{e}}\right)}\left(1-\frac{1}{1-\frac{\gamma_{m}}{\overline{\gamma_{e}}}+\frac{\gamma_{m}}{\overline{\gamma_{s e}}}}\right) e^{\frac{1-\frac{16}{\pi_{s e}^{2}}}{\bar{\gamma}_{s e}}}-\frac{\sqrt{\pi}}{2}(1-$

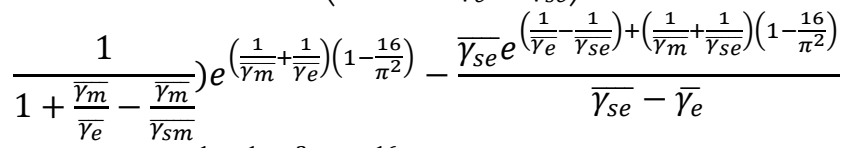
$-\frac{\sqrt{\pi} \cdot \overline{\gamma_{s e}} \cdot e^{\left(\frac{1}{\overline{\gamma_{m}}}-\frac{1}{\overline{\gamma_{e}}}+\frac{2}{\overline{\gamma_{s e}}}\right)\left(1-\frac{16}{\pi^{2}}\right)}}{2\left(\overline{\gamma_{s e}}-\overline{\gamma_{e}}\right)\left(1-\frac{\overline{\gamma_{m}}}{\overline{\gamma_{e}}}+\frac{\overline{\overline{\gamma_{m}}}}{\overline{\gamma_{s e}}}\right)}+\frac{\pi \rho_{X S}}{2\left(\overline{\gamma_{s m}}-\overline{\overline{\gamma_{m}}}\right)} e^{\frac{1}{2}\left(\frac{1}{\overline{\gamma_{m}}}+\frac{1}{\overline{\gamma_{s m}}}\right)}$ $. K_{-1}\left(\frac{1}{2}\left(\frac{1}{\overline{\gamma_{m}}}-\frac{1}{\overline{\gamma_{s m}}}\right)\right)-\frac{\pi}{2} \frac{\rho_{X s} e^{\frac{1}{2}\left(\frac{1}{\overline{\gamma_{e}}}+\frac{1}{\overline{\gamma_{s e}}}\right)}}{\left(\overline{\gamma_{s e}}-\overline{\gamma_{e}}\right)} K_{-1}\left(\frac{1}{2}\left(\frac{1}{\overline{\gamma_{e}}}-\frac{1}{\overline{\gamma_{s e}}}\right)\right)-$ $\frac{\pi}{2}\left(\frac{\rho_{X S} e^{\frac{1}{2}\left(\frac{1}{\overline{\gamma_{m}}}+\frac{1}{\overline{\gamma_{e}}}+\frac{1}{\overline{\gamma_{s m}}}\right)}}{\overline{\gamma_{s m}}+\frac{\overline{\gamma_{s m}} \cdot \overline{\gamma_{m}}}{\overline{\gamma_{e}}}-\overline{\overline{\gamma_{m}}}}\right) K_{-1}\left(\frac{\frac{1}{\overline{\gamma_{m}}}+\frac{1}{\overline{\gamma_{e}}}-\frac{1}{\overline{\gamma_{s m}}}}{2}\right)+\frac{2 \sqrt{\pi} \rho_{X S} e^{\frac{1}{\overline{\gamma_{s m}}}}}{\overline{\gamma_{s m}} \cdot \overline{\gamma_{m}} \sqrt{\overline{\gamma_{s m}}}}$ $\cdot\left[\frac{1}{\left(\frac{1}{\overline{\gamma_{m}}}-\frac{1}{\overline{\gamma_{s m}}}\right)^{\frac{3}{2}}}\left(\sum_{n=0}^{\infty} \frac{\left(\frac{-1}{\overline{\gamma_{s m}}}\right)^{n} \Gamma\left(n+\frac{5}{2}, 1-\frac{\overline{\gamma_{m}}}{\overline{\gamma_{s m}}}\right)}{n !(2 n+1)\left(\frac{1}{\overline{\gamma_{m}}}-\frac{1}{\overline{\gamma_{s m}}}\right)^{n+1}}\right.\right.$

$\left.+\sum_{n=0}^{\infty} \sum_{k=0}^{n} \frac{\left(\frac{-1}{\overline{\gamma_{s m}}}\right)^{n} \Gamma(n+2) \Gamma\left(k+\frac{3}{2}, 1-\frac{\overline{\overline{\gamma_{m}}}}{\overline{\gamma_{s m}}}\right)}{n !(2 n+1)\left(\frac{1}{\overline{\gamma_{m}}}-\frac{1}{\overline{\gamma_{s m}}}\right)^{k} \Gamma(k+1) \Gamma(n+2-k)}\right)$

$-\frac{1}{\left(\frac{1}{\overline{\gamma_{m}}}+\frac{1}{\overline{\gamma_{e}}}-\frac{1}{\overline{\gamma_{s m}}}\right)^{\frac{3}{2}}}\left(\sum_{n=0}^{\infty} \frac{\left(\frac{-1}{\overline{\gamma_{s m}}}\right)^{n} \Gamma\left(n+\frac{5}{2}, 1+\frac{\overline{\gamma_{m}}}{\overline{\gamma_{e}}}-\frac{\overline{\gamma_{m}}}{\overline{\gamma_{s m}}}\right)}{n !(2 n+1)\left(\frac{1}{\overline{\gamma_{m}}}+\frac{1}{\overline{\gamma_{e}}}-\frac{1}{\overline{\gamma_{s m}}}\right)^{n+1}}+\right.$

$\sum_{n=0}^{\infty} \sum_{k=0}^{n} \frac{\left(\frac{-1}{\overline{\gamma_{s m}}}\right)^{n} \Gamma(n+2) \Gamma\left(k+\frac{3}{2}, 1+\frac{\overline{\gamma_{m}}}{\overline{\gamma_{e}}}-\frac{\overline{\gamma_{m}}}{\overline{\gamma_{s m}}}\right)}{\left.n !(2 n+1) \Gamma(k+1) \Gamma(n+2-k)\left(\frac{1}{\overline{\gamma_{m}}}+\frac{1}{\overline{\gamma_{e}}}-\frac{1}{\overline{\gamma_{s m}}}\right)^{k}\right]}$

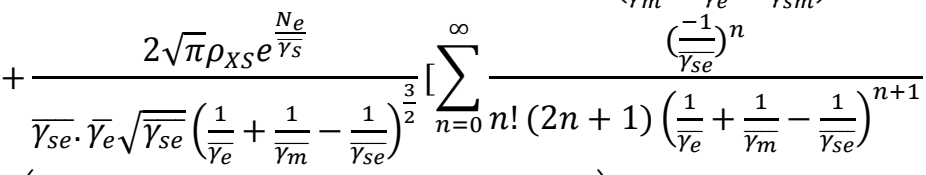

$\cdot\left(\Gamma\left(n+\frac{5}{2}, 1+\frac{\overline{\gamma_{m}}}{\overline{\gamma_{e}}}-\frac{\overline{\gamma_{m}}}{\overline{\gamma_{s e}}}\right)-\Gamma\left(n+\frac{5}{2}, 0\right)\right)+$

$\left.\sum_{n=0}^{\infty} \sum_{k=0}^{n} \frac{\left(\frac{-1}{\overline{\gamma_{s e}}}\right)^{n}\left(\begin{array}{c}n+1 \\ k\end{array}\right)\left(\Gamma\left(k+\frac{3}{2}, 1+\frac{\overline{\gamma_{m}}}{\overline{\gamma_{e}}}-\frac{\overline{\gamma_{m}}}{\overline{\gamma_{s e}}}\right)-\Gamma\left(k+\frac{3}{2}, 0\right)\right)}{n !(2 n+1)\left(\frac{1}{\overline{\gamma_{e}}}+\frac{1}{\overline{\gamma_{m}}}-\frac{1}{\overline{\gamma_{s e}}}\right)^{k}}\right]$.

Proof. The details of proof are in Appendix B.

Now, we are interested in obtaining secrecy outage probability (SOP), because it is an appropriate metric for evaluating security performance in communication systems and defined as a probability that the SC falls below a predefined secure transmission rate $R_{s}$. According to this definition, we can express SOP mathematically for Corollaries 1 and 2 as presented in below sub-section [7, 10, 16, 26].

SOP analysis. We want to analyze the SOP since it is an appropriate metric for appraising security performance in communication systems. This metric is defined as a probability that the SC falls under an already stated secure transmission rate $R_{S}>0$. According to this definition, the SOP is presented as

$$
P_{S O P}=P\left(C_{s} \leq R_{S}\right)=1-P\left(C_{s}>R_{S}\right)=1-P_{C},
$$

where $P_{C}$ is the complementary SOP and defined as

$$
P_{C}=P\left(C_{s}>R_{s}\right) \text {. }
$$

Theorem 4. The SOP for concerned uncorrelated Rayleigh fading WC with defined parameters $\overline{\gamma_{m}}, \overline{\gamma_{e}}, \overline{\gamma_{s m}}, \overline{\gamma_{s e}}$ and $R_{s}$ that the channel input $X$ is correlated to $S$ with coefficient $\rho_{X S}$, is given by

For Corollary 1:

For Corollary 2:

$$
P_{S O P}=1-\exp \left(\frac{1-2^{R_{S}}}{\left(1-\rho_{X S}^{2}\right) \overline{\gamma_{m}}}\right) .
$$


$=1-\frac{e^{\frac{2^{R_{S-1}}}{\overline{\gamma_{m}}}}}{A \sqrt{B^{3}}}\left[\frac{\overline{\gamma_{m}} \sqrt{B^{3}}+\overline{\gamma_{e}} 2^{R_{s}}}{\sqrt{\frac{A}{\overline{\gamma_{s e}}}}}-\frac{\frac{\pi 2^{R_{S}-1} \rho_{X S}}{\overline{\gamma_{m} \gamma_{s e} \gamma_{e}}}}{\overline{\gamma_{m} \gamma_{s e}} \overline{\gamma_{e}} \sqrt{\frac{A}{\overline{\gamma_{s e}}}}}-\frac{\sqrt{\pi}\left(2^{R_{s}} \rho_{X S}\right)}{2}-\right.$

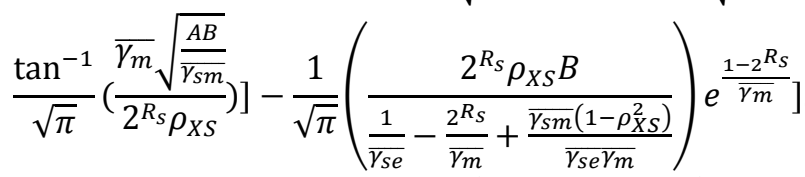

where $A=1-\frac{\overline{\gamma_{s e}} 2^{R_{S}}}{\overline{\gamma_{m}}}+\frac{\overline{\gamma_{S m}}\left(1-\rho_{X S}^{2}\right)}{\overline{\gamma_{m}}}-\frac{\overline{\gamma_{s e} \gamma_{e}} 2^{2 R_{S}} \rho_{X S}^{2}}{\overline{\gamma_{m}}\left(\overline{\gamma_{m}}+{\overline{\gamma_{e}}}^{2 R_{S}}\right)}$ and $B=$ $\frac{1}{\overline{\gamma_{e}}}+\frac{2^{R_{S}}}{\overline{\gamma_{m}}}$.

Proof. The details of proof are in Appendix C.

It is very important to examine the asymptotic behavior of SOP for extreme values of the target secrecy rate $R_{S}$. Specifically, for theorem 4 (that satisfies Corollary 1): when $R_{S} \rightarrow 0$ we have:

$$
P_{\text {sop }} \rightarrow 1-1=0 \text {, }
$$

and when $R_{s} \rightarrow \infty$, we have $P_{\text {sop }} \rightarrow 1$, meaning that secure transmission becomes impossible at very high rates. Also, for theorem 5 (that satisfies Corollary 2): when $R_{s} \rightarrow 0$ we have

$P_{\text {sop }} \rightarrow 1-\frac{1}{\left(\frac{1}{\overline{\gamma_{e}}}+\frac{1}{\overline{\gamma_{m}}}\right)^{\frac{3}{2}}\left(1-\frac{\overline{\gamma_{s e}}}{\overline{\gamma_{m}}}+\frac{\overline{\gamma_{s m}}\left(1-\rho_{X S}^{2}\right)}{\overline{\gamma_{m}}}-\frac{\overline{\gamma_{s e} \gamma_{e}} \rho_{X S}^{2}}{\overline{\gamma_{m}}\left(\overline{\gamma_{m}}+\overline{\gamma_{e}}\right)}\right)}$

$\cdot\left[\frac{\overline{\gamma_{m}}\left(\frac{1}{\overline{\gamma_{e}}}+\frac{1}{\overline{\gamma_{m}}}\right)^{\frac{3}{2}}}{\overline{\gamma_{m}}+\overline{\gamma_{e}}}-\frac{\frac{\pi \rho_{X S}}{2 \overline{\gamma_{m} \gamma_{s e} \gamma_{e}}}}{\sqrt{\frac{1}{\overline{\gamma_{s e}}}-\frac{1}{\overline{\gamma_{m}}}+\frac{\overline{\gamma_{s m}}\left(1-\rho_{X S}^{2}\right)}{\overline{\gamma_{s e} \gamma_{m}}}-\frac{\overline{\gamma_{e}} \rho_{X S}^{2}}{\overline{\gamma_{m}}\left(\overline{\gamma_{m}}+\overline{\gamma_{e}}\right)}}}\right.$

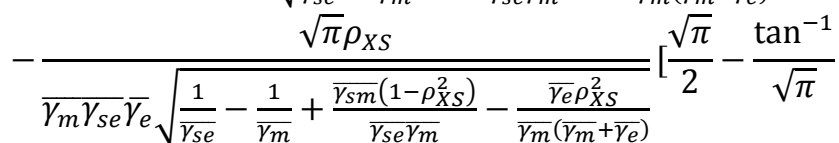

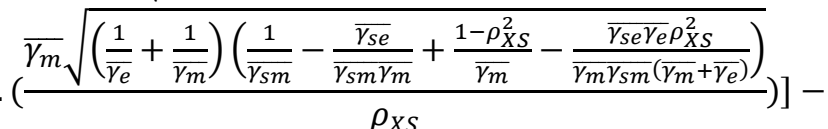

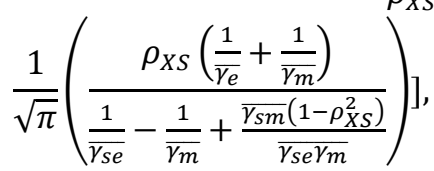

and when $R_{s} \rightarrow \infty$, we have $P_{\text {sop }} \rightarrow 1$, meaning that secure transmission becomes impossible at very high rates. We also investigate the performance of SOP in the high SNR regime (i.e. $\gamma_{m} \gg 1, \gamma_{e} \gg 1, \gamma_{s m} \gg 1$ and $\gamma_{s e} \gg 1$ ). Thus, the asymptotic complementary SOP is defined as follows:

For Corollary 1:

$$
\begin{aligned}
P_{C} & =P\left(\log \left(\left(1-\rho_{X S}^{2}\right) \gamma_{m}\right)>R_{s}\right) \\
& =\int_{0}^{\infty} \int_{\frac{{ }_{2} R_{S}}{1-\rho_{X S}^{2}}}^{\infty} f\left(\gamma_{m}, \gamma_{e}\right) d \gamma_{m} \cdot d \gamma_{e},
\end{aligned}
$$

where by calculating the integral of (20), the asymptotic SOP is given by

For Corollary 2:

$$
P_{S O P}^{a s y}=1-\exp \left(-\frac{2^{R_{S}}}{\left(1-\rho_{X S}^{2}\right) \overline{\gamma_{m}}}\right) \text {. }
$$

$$
\begin{aligned}
P_{C} & =P\left(\log \left(\frac{\gamma_{m}+\frac{\gamma_{s}}{N_{m}}+2 \rho_{X S} \sqrt{\gamma_{m} \cdot \frac{\gamma_{s}}{N_{m}}}}{\gamma_{e}+\frac{\gamma_{s}}{N_{e}}+2 \rho_{X S} \sqrt{\gamma_{e} \cdot \frac{\gamma_{s}}{N_{e}}}}\right)>R_{s}\right) \\
& <\int_{0}^{\infty} \int_{0}^{\infty} \int_{w^{\prime}}^{\infty} f\left(\gamma_{m}, \gamma_{s}, \gamma_{e}\right) d \gamma_{m} d \gamma_{e} d \gamma_{s},
\end{aligned}
$$

where $w^{\prime}=2^{R_{S}}\left(\gamma_{e}+\frac{\gamma_{s}}{N_{e}}+2 \rho_{X S} \sqrt{\gamma_{e} \cdot \frac{\gamma_{s}}{N_{e}}}\right)-\frac{\gamma_{s}\left(1-\rho_{X S}^{2}\right)}{N_{m}} . \quad$ By calculating the integral of (22) similarly proof of the theorem 4 , the asymptotic SOP is given by

$P_{\text {SOP }}^{\text {asy }}<$

$$
\begin{aligned}
& 1-\frac{1}{\left(1-\frac{\overline{\gamma_{s e}} 2^{R_{S}}}{\overline{\gamma_{m}}}+\frac{\overline{\gamma_{s m}}\left(1-\rho_{X S}^{2}\right)}{\overline{\gamma_{m}}}-\frac{\overline{\gamma_{s e} \gamma_{e}} 2^{2 R_{S}} \rho_{X S}^{2}}{\overline{\gamma_{m}}\left(\overline{\gamma_{m}}+\overline{\gamma_{e}} 2^{R_{S}}\right)}\right)\left(\frac{1}{\overline{\gamma_{e}}}+\frac{2^{R_{S}}}{\overline{\gamma_{m}}}\right)^{\frac{3}{2}}} \\
& \cdot\left[\frac{\overline{\gamma_{m}}\left(\frac{1}{\overline{\gamma_{e}}}+\frac{2^{R_{S}}}{\overline{\gamma_{m}}}\right)^{\frac{3}{2}}}{\overline{\gamma_{m}}+\overline{\gamma_{e}} 2^{R_{S}}}-\frac{\frac{\pi 2^{R_{S}-1} \rho_{X S}}{\overline{\gamma_{m} \gamma_{s e} \gamma_{e}}}}{\sqrt{\frac{1}{\overline{\gamma_{s e}}}-\frac{2^{R_{S}}}{\overline{\gamma_{m}}}+\frac{\overline{\gamma_{s m}}\left(1-\rho_{X S}^{2}\right)}{\overline{\gamma_{s e} \gamma_{m}}}-\frac{\overline{\gamma_{e}} 2^{2 R_{S} \rho_{X S}^{2}}}{\overline{\gamma_{m}}\left(\overline{\gamma_{m}}+\overline{\gamma_{e}} 2^{R_{S}}\right)}}}\right.
\end{aligned}
$$

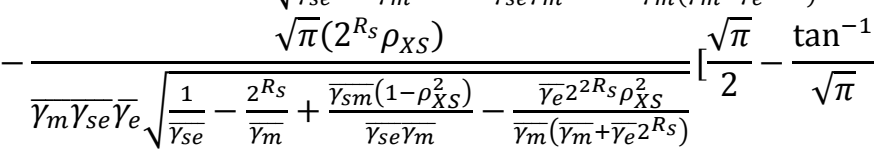

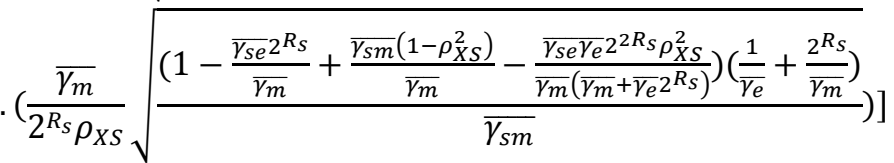

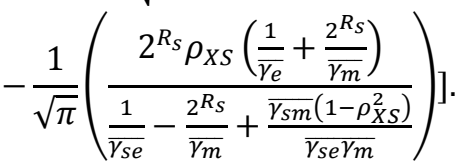

\section{NUMERICAL RESULTS}

In this section, the numerical results have been presented to demonstrate impact of correlation between the channel input and the SI on the physical layer security performances of the wireless WC. The results for the WCs satisfying Corollary 1 are omitted for simplicity and only, we illustrated these results for WCs satisfying Corollary 2 conditions.

The impact of the $\rho_{X S}$ on the ASC based on the variations of $\overline{\gamma_{m}}$ is illustrated in Fig. 2. It can be seen that $C_{s}^{a v g}$ decreases by increasing $\rho_{X S}$ for a given value of $\overline{\gamma_{m}}$. As, we can see, for high the main channel average SNR $\overline{\gamma_{m}}$, the impact of $\rho_{X S}$ on the $C_{s}^{a v g}$ is reduced. 


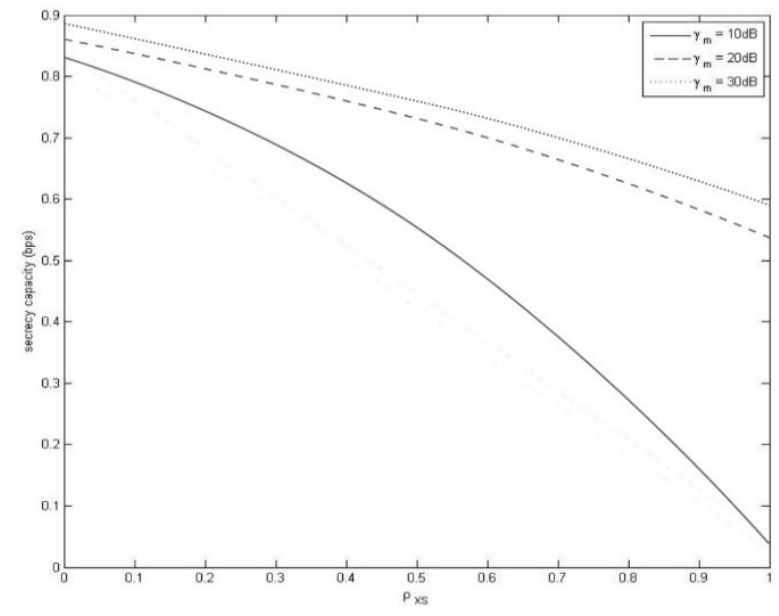

Fig. 2. The ASC versus $\overline{\gamma_{m}}$, for different values of $\rho_{X S}$.

In Fig. 3, the behavior of SOP based on variations of $\overline{\gamma_{m}}$ for selected values of parameter $\rho_{X S}$, and a target secrecy rate $R_{S}=$ 0.1 bits is illustrated. It is clearly seen that SOP constantly increases by increasing $\rho_{X S}$ for a specified value of $\overline{\gamma_{m}}$.

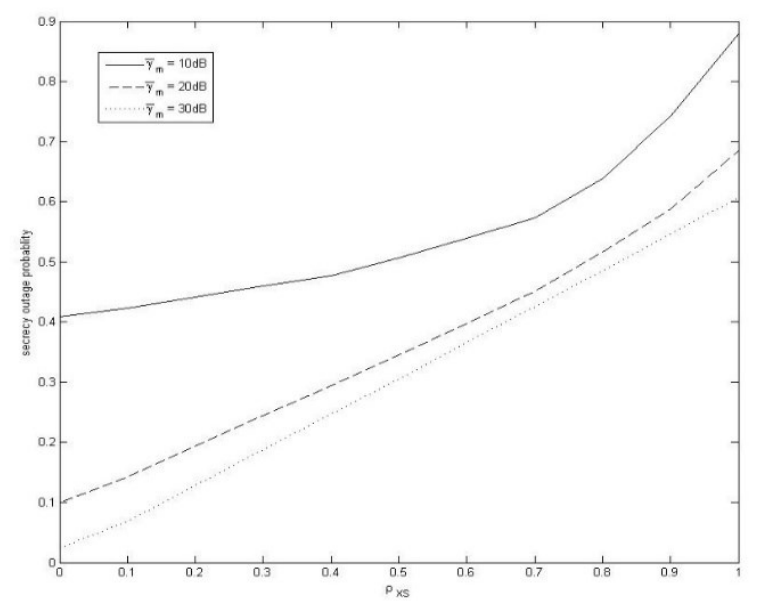

Fig. 3. SOP versus $\overline{\gamma_{m}}$, for different values of $\rho_{X S}$ and for a target secrecy rate $R_{s}=0.1$ bits.

\section{CONCLUSIONS}

In this work, the performance of physical layer security in a Rayleigh fading WC with considering side information noncausally available at the transmitter by considering the correlation between the channel input and the SI was analyzed. We demonstrated the impact of correlation between the channel input and the SI, by deriving closed-form expressions, on the average SC and secrecy outage probability as remarkable wireless performance factor for a wireless WC. Further, by Mathematical derivations and numerical results, it was investigated that correlation between channel input and SI has positive effects over ASC and SOP.

\section{Appendices}

Proof of Theorem 1: An informal proof of this theorem is a straightforward algebra extension of [5], [22], and [23] with some differences caused by known fading coefficients. The coding scheme used to achieve this capacity is similar to the one used in [18], [22], [23], [34], and [35]. The coding scheme and error probability analysis is straightforward and is not shown here due to a lack of space. We use definitions in corollary 1 and 2 to compute the capacity of WC with knowing SI at the transmitter and fading coefficients. We have following assumptions:

$$
\begin{aligned}
& E\left[X^{2}\right]=P, E\left[S^{2}\right]=Q, E\left[\eta_{m}^{2}\right]=N_{m}, E\left[\eta_{e}^{2}\right]=N_{e}, \\
& E(X, S)=\rho_{X S} \sqrt{P Q},
\end{aligned}
$$

and $U_{m}$ and $U_{e}$ are generated by using generalized dirty paper coding presented in [34]. According to [5], [22], [23], [34] and knowing that $Y_{m}=h_{m} X+h_{s m} S+\eta_{m}, Y_{e}=h_{e} X+h_{s e} S+\eta_{e}$ we can write mutual information related to the term $C_{m}$ in corollary 1 as follows:

$$
\begin{aligned}
& I\left(U_{m} ; Y_{m}\right) \\
& =H\left(h_{m} X+h_{s m} S+\eta_{m}\right)-H\left(h_{m} X+h_{s m} S+\eta_{m} \mid X+\alpha_{m} S\right) \\
& =H\left(h_{m} X+h_{s m} S+\eta_{m}\right)+H\left(X+\alpha_{m} S\right) \\
& -H\left(h_{m} X+h_{s m} S+\eta_{m}, X+\alpha_{m} S\right)= \\
& \log (2 \pi e)\left(\left|h_{m}\right|^{2} P+\left|h_{s m}\right|^{2} Q+N_{m}+2\left|h_{m}\right|\left|h_{s m}\right| \rho_{X S} \sqrt{P Q}\right) \\
& \quad+\log (2 \pi e)\left(P+\alpha_{m}^{2} Q+2 \alpha_{m} \rho_{X S} \sqrt{P Q}\right)- \\
& \log \left((2 \pi e)^{2} \operatorname{det}\left(\operatorname{cov}\left(h_{m} X+h_{s m} S+\eta_{m}, X+\alpha_{m} S\right)\right)\right)= \\
& \log \left(\frac{\left(\left|h_{m}\right|^{2} P+\left|h_{s m}\right|^{2} Q+N_{m}+2\left|h_{m}\right|\left|h_{s m}\right| \rho_{X S} \sqrt{P Q}\right)\left(P+\alpha_{m}^{2} Q+2 \alpha_{m} \rho_{X S} \sqrt{P Q}\right)}{P Q\left(1-\rho_{X S}^{2}\right)\left(\left|h_{s m}\right|-\alpha_{m}\left|h_{m}\right|\right)^{2}+N_{m}\left(P+\alpha_{m}^{2} Q+2 \alpha_{m} \rho_{X S} \sqrt{P Q}\right)}\right)
\end{aligned}
$$$$
\text { , }
$$

and

$$
I\left(U_{m} ; S\right)=\log \left(\frac{P+\alpha_{m}^{2} Q+2 \alpha_{m} \rho_{X S} \sqrt{P Q}}{P\left(1-\rho_{X S}^{2}\right)}\right) .
$$

Substituting (25) and (26 in $C_{m}$ with corollary 1, we obtain: $C_{m}\left(\alpha_{m}\right)=$ $\log \left(\frac{P\left(1-\rho_{X S}^{2}\right)\left(\left|h_{m}\right|^{2} P+\left|h_{s m}\right|^{2} Q+N_{m}+2\left|h_{m}\right|\left|h_{S m}\right| \rho_{X S} \sqrt{P Q}\right)}{P Q\left(1-\rho_{X S}^{2}\right)\left(\left|h_{s m}\right|-\alpha_{m}\left|h_{m}\right|\right)^{2}+N_{m}\left(P+\alpha_{m}^{2} Q+2 \alpha_{m} \rho_{X S} \sqrt{P Q}\right)}\right)$.

By maximizing $C_{m}\left(\alpha_{m}\right)$ over $\alpha_{m}$, for corollary 1, we get

$$
\begin{aligned}
C_{S} & =\max _{\alpha_{m}} C_{m}\left(\alpha_{m}\right)=\log \left(1+\frac{\left|h_{m}\right|^{2} P\left(1-\rho_{X S}^{2}\right)}{N_{m}}\right) \\
& =\log \left(1+\left(1-\rho_{X S}^{2}\right) \gamma_{m}\right) .
\end{aligned}
$$

So we have (13) for corollary 1 . The proof of this theorem for corollary 2 is similar with negligible change and is omitted for the lack of space.

\section{Appendix B:}

Proof of Theorem 2: First, we state some integrals that are needed to prove the theorems in [30] and [31]. Therefore, we have

$$
\begin{aligned}
& \int e^{-\xi t} \log (1+\beta t) d t \\
& =\frac{1}{\xi}\left[e^{\frac{\xi}{\beta}} E i\left(-\left(\xi t+\frac{\xi}{\beta}\right)\right)-e^{-\xi t} \log (1+\beta t)\right]
\end{aligned}
$$

\section{Appendix A:}


$\int_{0}^{\infty} e^{-\xi t} \log (1+\beta t) d t=-\frac{e^{\frac{\xi}{\beta}}}{\xi} E i\left(-\frac{\xi}{\beta}\right)$

$\int e^{-v x} E i(-(\delta+\kappa x)) d x=$

$\frac{1}{v}\left[e^{\frac{v \delta}{\kappa}} E i\left(-\frac{(v+\kappa)(\kappa x+\delta)}{k}\right)-e^{v x} E i(-(\kappa x+\delta))\right]$,

$\int_{0}^{\infty} e^{-v x} \operatorname{Ei}(-(\delta+\kappa x)) d x$

$=\frac{1}{v}\left[E i(-\delta)-e^{\frac{v \delta}{\kappa}} E i\left(-\frac{(v+\kappa) \delta}{k}\right)\right]$,

$\int_{0}^{\infty} \frac{t^{v-1}}{t+\beta} e^{-\xi t} d t=\beta^{v-1} e^{\beta \xi} \Gamma(v) \Gamma(1-\mathrm{v}, \beta \xi)$,

$\int_{0}^{\infty} e^{-\xi t} \log (\beta+t) d t=\frac{1}{\xi}\left[\ln \beta-e^{\xi \beta} E i(-\xi \beta)\right]$,

$\int_{0}^{\infty} t^{v-1}(t+\beta)^{v-1} e^{-\xi t} d t=\frac{\left(\frac{\beta}{\xi}\right)^{v-\frac{1}{2}} e^{\frac{\xi \beta}{2}} \Gamma(v) K_{\frac{1}{2}-V}\left(\frac{\xi \beta}{2}\right)}{\sqrt{\pi}}$.

$\int e^{-\xi t+\beta \sqrt{t}} d t=-\frac{e^{-\xi t+\beta \sqrt{t}}}{\xi}-\frac{\beta \sqrt{\pi} e^{\frac{\beta^{2}}{4 \xi}} \operatorname{erf}\left(-\sqrt{\xi t}+\frac{\beta}{2 \sqrt{\xi}}\right)}{2 \xi^{\frac{3}{2}}}$,

$\int \sqrt{t} e^{-\xi t} d t=-\frac{\sqrt{t} e^{-\xi t}}{\xi}+\frac{\sqrt{\pi} \operatorname{erf}(\sqrt{\xi t})}{2 \xi^{\frac{3}{2}}}$,

$\int_{0}^{\infty} t^{2} e^{-\xi^{2} t^{2}} \operatorname{erf}(\beta t) d t$

$=\frac{\sqrt{\pi}}{4 \xi^{3}}-\frac{1}{2 \sqrt{\pi}}\left[\frac{\tan ^{-1}\left(\frac{\xi}{\beta}\right)}{\xi^{3}}-\frac{\beta}{\xi^{2}\left(\beta^{2}+\xi^{2}\right)}\right]$,

where $E i(x)=-\int_{x}^{\infty} t^{-1} e^{-t} d t$. Also, $E i(-x)$ was approximated in [32] as follows:

$$
E i(-x)=-4 \sqrt{2} a_{N} a_{I} \sum_{n=1}^{N+1} \sum_{i=1}^{I+1} \sqrt{b_{n}} e^{-4 b_{n} b_{i} x},
$$

where $\theta_{0}=0<\theta_{1}<\cdots<\theta_{N+1}=\frac{\pi}{2}, a_{N}=\frac{\theta_{n}-\theta_{n-1}}{\pi}, b_{n}=$ $\frac{\cos \left(\theta_{n-1}\right)-\cos \left(\theta_{n}\right)}{2\left(\theta_{n}-\theta_{n-1}\right)}$, and for $N=I=1$ we have: $a_{N}=a_{I}=\frac{1}{4}$, $b_{1}=\infty$ and $b_{2}=\frac{2}{\pi}$. Thus, $\operatorname{Ei}(-x)$ can be approximated as

$$
E i(-x) \sim-\frac{\sqrt{\pi}}{2} e^{-\left(\frac{16}{\pi^{2}}\right) x} .
$$

Also, $K_{V}(x)=\frac{2}{\pi} \int_{0}^{\frac{\pi}{2}} \cos (x \tan \theta-n \theta) d \theta$ is the Bateman function studied in [33].

Thus, utilizing linear formulas of integration, (13-Corollary 1) can be decomposed as

$$
\begin{aligned}
C_{s}^{a v g} & =\int_{0}^{\infty} \int_{0}^{\infty} \log \left(1+\left(1-\rho_{X S}^{2}\right) \gamma_{m}\right) f\left(\gamma_{m}, \gamma_{e}\right) d \gamma_{m} \cdot d \gamma_{e} \\
& =\int_{0}^{\infty} \frac{1}{\overline{\bar{\gamma}}} e^{-\frac{\gamma_{e}}{\overline{\gamma_{e}}}} d \gamma_{e} \cdot \int_{0}^{\infty} \log \left(1+\left(1-\rho_{X S}^{2}\right) \gamma_{m}\right) \frac{1}{\overline{\gamma_{m}}} e^{-\frac{\gamma_{m}}{\overline{\gamma_{m}}}} d \gamma_{m} \\
& =-e^{\overline{\left(1-\rho_{X S}^{2}\right) \overline{\overline{\gamma m}}}} E i\left(-\frac{1}{\left(1-\rho_{X S}^{2}\right) \overline{\gamma_{m}}}\right) .
\end{aligned}
$$

where (41) is exploiting from (30). Eventually, by considering the obtained approximation in (40), the proof for (14) is completed. For Corollary 2, utilizing linear formulas of integration, (13-Corollary 2) can be decomposed as

$$
C_{s}^{a v g}=C_{1}-C_{2} \text {, }
$$

where

$$
\begin{gathered}
C_{1}=\int_{0}^{\infty} \int_{0}^{\gamma_{m}} \int_{0}^{\infty} \log \left(1+\gamma_{m}+\gamma_{s m}+2 \rho_{X S} \sqrt{\gamma_{m} \cdot \gamma_{s m}}\right) \\
. f\left(\gamma_{s m}, \gamma_{m}, \gamma_{e}\right) d \gamma_{s m} d \gamma_{e} d \gamma_{m},
\end{gathered}
$$

and

$$
C_{2}=\int_{0}^{\infty} \int_{0}^{\gamma_{m}} \int_{0}^{\infty} \log \left(1+\gamma_{e}+\gamma_{s e}+2 \rho_{X s} \sqrt{\gamma_{e} \cdot \gamma_{s e}}\right)
$$

By utilizing linear formulas of integration, for the first term of Eq. (42), we receive to

$C_{1}=$

$\int_{0}^{\infty} \int_{\gamma_{\gamma_{m}}}^{\gamma_{m}} \frac{e^{-\left(\frac{\gamma_{m}}{\gamma_{m}}+\frac{\gamma_{e}}{\overline{\gamma_{e}}}\right)}}{\overline{\gamma_{s m}} \overline{\gamma_{e}} \overline{\gamma_{m}}}\left(\int_{0}^{\infty} \log \left(1+\gamma_{m}+\gamma_{s m}+2 \rho_{X s} \sqrt{\gamma_{m} \cdot \gamma_{s m}}\right)\right.$

.$\left.e^{-\frac{\gamma_{s m}}{\gamma_{s m}}} d \gamma_{s m}\right) d \gamma_{e} d \gamma_{m}$.

We define $C_{1}{ }^{\prime}$ as below

$C_{1}{ }^{\prime}=\int_{0}^{\infty} \log \left(1+\gamma_{m}+\gamma_{s m}+2 \rho_{X s} \sqrt{\gamma_{m} \gamma_{s m}}\right) e^{-\frac{\gamma_{s m}}{\gamma_{s m}}} d \gamma_{s m}$

where by using the properties of logarithm, we have

$$
\begin{aligned}
& C_{1}{ }^{\prime}=\int_{0}^{\infty} \log \left(1+\gamma_{m}+\gamma_{s m}\right) e^{-\frac{\gamma_{s m}}{\gamma_{s m}}} d \gamma_{s m}+ \\
& \quad \int_{0}^{\infty} \log \left(1+\frac{2 \rho_{X S} \sqrt{\gamma_{m} \gamma_{s m}}}{1+\gamma_{m}+\gamma_{s m}}\right) e^{-\frac{\gamma_{s m}}{\gamma_{s m}}} d \gamma_{s m}=w_{1}+w_{2},
\end{aligned}
$$

The logarithmic functions, when $x \ll 1$, can be approximated as follows

$$
\log (1+x) \cong x .
$$

So, by exploiting from (33) and (34), we have

$$
\begin{aligned}
w_{1} & =\int_{0}^{\infty} \log \left(1+\gamma_{m}+\gamma_{s m}\right) e^{-\frac{\gamma_{s m}}{\gamma_{s m}}} d \gamma_{s m} \\
& =\overline{\gamma_{s m}}\left[\ln \left(1+\gamma_{m}\right)-e^{\frac{1+\gamma_{m}}{\overline{\gamma_{s m}}}} E i\left(-\frac{1+\gamma_{m}}{\overline{\gamma_{s m}}}\right),\right. \\
w_{2} & =\int_{0}^{\infty} \frac{2 \rho_{X S} \sqrt{\gamma_{m} \gamma_{s m}}}{1+\gamma_{m}+\gamma_{s m}} e^{-\frac{\gamma_{s m}}{\gamma_{s m}}} d \gamma_{s m}=2 \rho_{X s} \sqrt{\gamma_{m}\left(1+\gamma_{m}\right)} \\
& . e^{\frac{1+\gamma_{m}}{\overline{\gamma_{s m}}}} \Gamma\left(\frac{3}{2}\right) \Gamma\left(\frac{1}{2}, \frac{1+\gamma_{m}}{\overline{\gamma_{s m}}}\right)=\pi \rho_{X s} \sqrt{\gamma_{m}\left(1+\gamma_{m}\right)} e^{\frac{1+\gamma_{m}}{\bar{\gamma}_{s m}}} \\
. & \left(1-\operatorname{erf}\left(\sqrt{\frac{1+\gamma_{m}}{\overline{\gamma_{s m}}}}\right)\right) .
\end{aligned}
$$

Now, we define $C_{1}{ }^{\prime \prime}$ as

$$
C_{1}^{\prime \prime}=\int_{0}^{\gamma_{m}} e^{-\frac{\gamma_{e}}{\gamma_{e}}}\left(C_{1}{ }^{\prime}\right) d \gamma_{e} .
$$

By replacing from (45), we have

$$
\begin{aligned}
C_{1}^{\prime \prime}= & \int_{0}^{\gamma_{m}} e^{-\frac{\gamma_{e}}{\overline{\gamma_{e}}}}\left[\overline{\gamma_{s m}} \ln \left(1+\gamma_{m}\right)-\overline{\gamma_{s m}} e^{\frac{1+\gamma_{m}}{\overline{\gamma_{s m}}}} E i\left(-\frac{1+\gamma_{m}}{\overline{\gamma_{s m}}}\right)+\right. \\
& \pi \rho_{X s} \sqrt{\gamma_{m}\left(1+\gamma_{m}\right)} e^{\frac{1+\gamma_{m}}{\bar{\gamma}_{s m}}}\left(1-\operatorname{erf}\left(\sqrt{\frac{1+\gamma_{m}}{\overline{\gamma_{s m}}}}\right)\right] d \gamma_{e} \\
= & j_{1}+j_{2}+j_{3}+j_{4},
\end{aligned}
$$


where, by exploiting from (29) and (31), we have $j_{1}=\int_{0}^{\gamma_{m}} \overline{\gamma_{s m}} e^{-\frac{\gamma_{e}}{\gamma_{e}}} \ln \left(1+\gamma_{m}\right) d \gamma_{e}=\overline{\gamma_{s m}} \overline{\gamma_{e}} \ln \left(1+\gamma_{m}\right)(1-$ $\left.e^{-\frac{\gamma m}{\overline{\gamma e}}}\right)$

and

$j_{2}=-\int_{0}^{\gamma_{m}} \overline{\gamma_{s m}} e^{-\frac{\gamma_{e}}{\overline{\gamma_{e}}}} e^{\frac{1+\gamma_{m}}{\overline{\gamma_{s m}}}} E i\left(-\frac{1+\gamma_{m}}{\overline{\gamma_{s m}}}\right) d \gamma_{e}=-\overline{\gamma_{s m}} \overline{\gamma_{e}}$

$\cdot e^{\frac{1+\gamma_{m}}{\overline{\gamma_{s m}}}}\left(1-e^{-\frac{\gamma_{m}}{\overline{\gamma_{e}}}}\right) E i\left(-\frac{1+\gamma_{m}}{\overline{\gamma_{s m}}}\right)$,

$j_{3}=\int_{0}^{\gamma_{m}} e^{-\frac{\gamma_{e}}{\gamma_{e}}}\left[\pi \rho_{X s} \sqrt{\gamma_{m}\left(1+\gamma_{m}\right)} e^{\frac{1+\gamma_{m}}{\gamma_{s m}}}\right] d \gamma_{e}=$

$\overline{\gamma_{e}}\left(1-e^{-\frac{\gamma_{m}}{\overline{\gamma_{e}}}}\right)\left(\pi \rho_{X S} \sqrt{\gamma_{m}\left(1+\gamma_{m}\right)} e^{\frac{1+\gamma_{m}}{\overline{\gamma_{s m}}}}\right)$,

$j_{4}=-\int_{0}^{\gamma_{m}} e^{-\frac{\gamma_{e}}{\gamma_{e}}}\left[\pi \rho_{X s} \sqrt{\gamma_{m}\left(1+\gamma_{m}\right)} e^{\frac{1+\gamma_{m}}{\overline{\gamma_{s m}}}} \operatorname{erf}\left(\sqrt{\frac{1+\gamma_{m}}{\overline{\gamma_{s m}}}}\right)\right]$ $d \gamma_{e}=-\overline{\gamma_{e}}\left(1-e^{-\frac{\gamma_{m}}{\overline{\gamma_{e}}}}\right)\left[\pi \rho_{X S} \sqrt{\gamma_{m}\left(1+\gamma_{m}\right)} e^{\frac{1+\gamma_{m}}{\overline{\gamma_{s} m}}}\right.$

.erf $\left.\left(\sqrt{\frac{1+\gamma_{m}}{\overline{\gamma_{s m}}}}\right)\right]$

Thus

$C_{1}=\int_{0}^{\infty} \frac{e^{-\frac{\gamma_{m}}{\gamma_{m}}}}{\overline{\gamma_{s m}} \cdot \overline{\gamma_{e}} \cdot \overline{\gamma_{m}}}\left(j_{1}+j_{2}+j_{3}+j_{4}\right) d \gamma_{m}=\sum_{i=1}^{8} k_{i}$,

where, by exploiting (29-35), we have

$$
\begin{aligned}
k_{1}= & \int_{0}^{\infty} \frac{e^{-\frac{\gamma_{m}}{\gamma_{m}}}}{\overline{\gamma_{m}}} \ln \left(1+\gamma_{m}\right) d \gamma_{m}=-e^{\frac{1}{\overline{\gamma m}}} E i\left(\frac{-1}{\overline{\gamma_{m}}}\right), \\
k_{2}= & \int_{0}^{\infty} \frac{e^{-\gamma_{m}\left(\frac{1}{\overline{\gamma m}}+\frac{1}{\overline{\gamma_{e}}}\right)}}{\overline{\gamma_{m}}} \ln \left(1+\gamma_{m}\right) d \gamma_{m}=-\frac{e^{\frac{1}{\gamma_{m}}+\frac{1}{\overline{\gamma e}}}}{\left(\frac{\overline{\gamma_{m}}}{\overline{\gamma_{e}}}+1\right)} \\
& . E i\left(-\left(\frac{1}{\overline{\gamma_{m}}}+\frac{1}{\overline{\gamma_{e}}}\right)\right),
\end{aligned}
$$$$
k_{3}=-\int_{0}^{\infty} \frac{e^{-\frac{\gamma_{m}}{\gamma_{m}}} e^{\frac{1+\gamma_{m}}{\overline{\gamma_{s m}}}}}{\overline{\gamma_{m}}} E i\left(-\frac{1+\gamma_{m}}{\overline{\gamma_{s m}}}\right) d \gamma_{m}
$$$$
=\frac{-\overline{\gamma_{s m}}}{\overline{\gamma_{s m}}-\overline{\gamma_{m}}} e^{\frac{1}{\overline{\gamma_{s m}}}}\left(E i\left(\frac{-1}{\overline{\gamma_{s m}}}\right)-e^{\frac{1}{\overline{\gamma_{m}}}-\frac{1}{\overline{\gamma_{s m}}}} E i\left(\frac{-1}{\overline{\gamma_{m}}}\right)\right) \text {, }
$$

$k_{4}=\int_{0}^{\infty} \frac{e^{-\gamma_{m}\left(\frac{1}{\overline{\gamma_{m}}}+\frac{1}{\bar{\gamma}_{e}}\right)} e^{\frac{1+\gamma_{m}}{\gamma_{s m}}}}{\overline{\gamma_{m}}} E i\left(-\frac{1+\gamma_{m}}{\overline{\gamma_{s m}}}\right) d \gamma_{m}=$

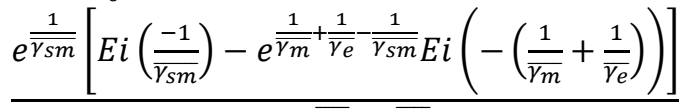

$$
1+\frac{\overline{\gamma_{m}}}{\overline{\gamma_{e}}}-\frac{\overline{\gamma_{m}}}{\overline{\gamma_{s m}}}
$$

$$
\begin{aligned}
& k_{5}=\int_{0}^{\infty} \frac{e^{-\frac{\gamma_{m}}{\gamma_{m}}}}{\overline{\gamma_{s m}} \cdot \overline{\gamma_{m}}}\left[\pi \rho_{X S} \sqrt{\gamma_{m}\left(1+\gamma_{m}\right)} e^{\frac{1+\gamma_{m}}{\overline{\gamma_{s m}}}}\right] d \gamma_{m} \\
& =\frac{\pi \rho_{X S} e^{\frac{\gamma_{m}}{\overline{\gamma_{s}}}}}{\overline{\gamma_{s m}} \cdot \overline{\gamma_{m}}} \int_{0}^{\infty} \sqrt{\gamma_{m}\left(1+\gamma_{m}\right)} e^{-\gamma_{m}\left(\frac{1}{\overline{\gamma_{m}}}-\frac{1}{\bar{\gamma}_{s m}}\right)} d \gamma_{m} \\
& =\frac{\pi}{2}\left(\frac{\rho_{X S}}{\overline{\gamma_{s m}}-\overline{\overline{\gamma_{m}}}}\right) e^{\frac{1}{2}\left(\frac{1}{\overline{\gamma_{m}}}+\frac{1}{\bar{\gamma}_{s m}}\right)} K_{-1}\left(\frac{1}{2}\left(\frac{1}{\overline{\gamma_{m}}}-\frac{1}{\overline{\gamma_{s m}}}\right)\right) \text {, }
\end{aligned}
$$

$$
\begin{aligned}
& k_{6}=-\int_{0}^{\infty} \frac{e^{-\frac{\gamma_{m}}{\gamma_{m}}} e^{-\frac{\gamma_{m}}{\bar{\gamma}}}}{\overline{\gamma_{s m}} \cdot \overline{\gamma_{m}}}\left[\pi \rho_{X S} \sqrt{\gamma_{m}\left(1+\gamma_{m}\right)} e^{\frac{1+\gamma_{m}}{\overline{\gamma_{s m}}}}\right] d \gamma_{m} \\
& =-\frac{\pi \rho_{X S} e^{\frac{1}{\gamma_{s m}}}}{\overline{\gamma_{s m}} \cdot \overline{\gamma_{m}}} \int_{0}^{\infty} \sqrt{\gamma_{m}\left(1+\gamma_{m}\right)} e^{-\gamma_{m}\left(\frac{1}{\gamma_{m}}+\frac{1}{\bar{\gamma}}-\frac{1}{\gamma_{s m}}\right)} d \gamma_{m}=
\end{aligned}
$$

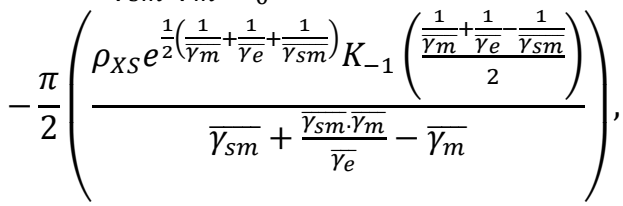

$. d \gamma_{m}=-\frac{\pi \rho_{X S} e^{\frac{1}{\overline{\gamma_{s m}}}}}{\overline{\gamma_{s m}} \cdot \overline{\gamma_{m}}} \int_{0}^{\infty} \sqrt{\gamma_{m}\left(1+\gamma_{m}\right)} e^{-\gamma_{m}\left(\frac{1}{\overline{\gamma_{m}}}-\frac{1}{\gamma_{s m}}\right)}$

$\operatorname{erf}\left(\sqrt{\frac{1+\gamma_{m}}{\overline{\gamma_{s m}}}}\right) d \gamma_{m}=\frac{-\pi \rho_{X S} e^{\frac{1}{\gamma_{s m}}}}{\overline{\gamma_{s m}} \cdot \overline{\gamma_{m}}} \int_{0}^{\infty} \sqrt{\gamma_{m}\left(1+\gamma_{m}\right)} \cdot \frac{2}{\sqrt{\pi}}$

$\sum_{n=0}^{\infty} \frac{(-1)^{n}\left(\sqrt{\frac{1+\gamma_{m}}{\overline{\gamma_{s m}}}}\right)^{2 n+1}}{n !(2 n+1)} e^{-\gamma_{m}\left(\frac{1}{\overline{\gamma_{m}}}-\frac{1}{\overline{\gamma_{s m}}}\right)} d \gamma_{m}=-\frac{2 \sqrt{\pi} \rho_{X S} e^{\frac{1}{\overline{\gamma_{s m}}}}}{\overline{\gamma_{s m}} \cdot \overline{\gamma_{m}}}$

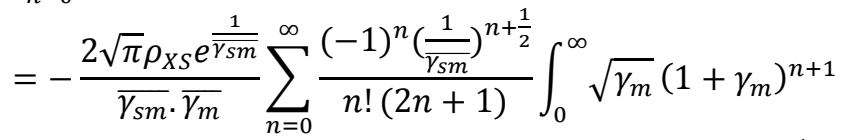

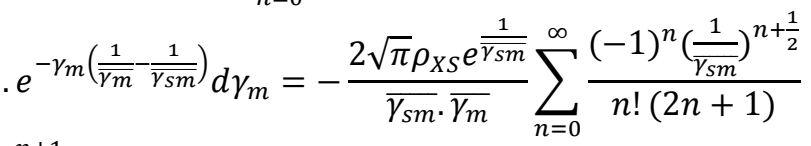

$\sum_{k=0}^{n+1}\left(\begin{array}{c}n+1 \\ k\end{array}\right) \int_{0}^{\infty} \gamma_{m}{ }^{k+\frac{1}{2}} \cdot e^{-\gamma_{m}\left(\frac{1}{\gamma_{m}}-\frac{1}{\gamma_{s m}}\right)} d \gamma_{m}$

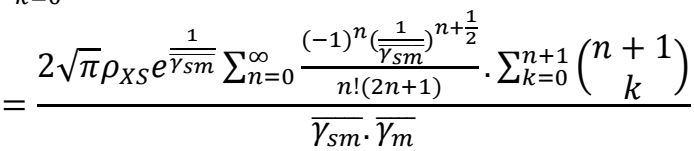

$\cdot\left(\frac{1}{\overline{\gamma_{m}}}-\frac{1}{\overline{\gamma_{s m}}}\right)^{-k-\frac{3}{2}} \Gamma\left(k+\frac{3}{2}, 1-\frac{\overline{\gamma_{m}}}{\overline{\gamma_{s m}}}\right)=\frac{2 \sqrt{\pi} \rho_{X s} e^{\frac{1}{\bar{\gamma}_{s m}}}}{\overline{\gamma_{s m}} \cdot \overline{\gamma_{m}}}$

$\sum_{n=0}^{\infty} \frac{(-1)^{n}\left(\frac{1}{\overline{\gamma_{s m}}}\right)^{n+\frac{1}{2}}}{n !(2 n+1)}\left(\left(\frac{1}{\overline{\gamma_{m}}}-\frac{1}{\overline{\gamma_{s m}}}\right)^{-n-\frac{5}{2}} \Gamma\left(n+\frac{5}{2}, 1-\frac{\overline{\gamma_{m}}}{\overline{\gamma_{s m}}}\right)\right.$

$\left.+\Gamma(n+2) . \sum_{k=0}^{n} \frac{\left(\frac{1}{\overline{\gamma_{m}}}-\frac{1}{\overline{\gamma_{s m}}}\right)^{-k-\frac{3}{2}} \Gamma\left(k+\frac{3}{2}, 1-\frac{\overline{\gamma_{m}}}{\overline{\gamma_{s m}}}\right)}{\Gamma(k+1) \Gamma(n+2-k)}\right)=$

$\frac{2 \sqrt{\pi} \rho_{X S} e^{\frac{1}{\overline{\gamma_{s m}}}}}{\overline{\gamma_{s m}} \cdot \overline{\gamma_{m}}}\left[\sum_{n=0}^{\infty} \frac{(-1)^{n}\left(\frac{1}{\overline{\gamma_{s m}}}\right)^{n+\frac{1}{2}}\left(\frac{1}{\overline{\gamma_{m}}}-\frac{1}{\overline{\gamma_{s m}}}\right)^{-n-\frac{5}{2}}}{n !(2 n+1)}\right.$

$. \Gamma\left(n+\frac{5}{2}, 1-\frac{\overline{\gamma_{m}}}{\overline{\gamma_{s m}}}\right)+\sum_{n=0}^{\infty} \frac{(-1)^{n}\left(\frac{1}{\overline{\gamma_{s m}}}\right)^{\left(n+\frac{1}{2}\right)} \Gamma(n+2)}{n !(2 n+1)}$

$\left.\sum_{k=0}^{n} \frac{\left(\frac{1}{\overline{\gamma_{m}}}-\frac{1}{\overline{\gamma_{s m}}}\right)^{-k-\frac{3}{2}} \Gamma\left(k+\frac{3}{2}, 1-\frac{\overline{\gamma_{m}}}{\overline{\gamma_{s m}}}\right)}{\Gamma(k+1) \Gamma(n+2-k)}\right]$ 
$k_{8}=$

$\int_{0}^{\infty} \frac{e^{-\frac{\gamma_{m}}{\overline{\gamma_{m}}}} e^{-\frac{\gamma_{m}}{\overline{\gamma_{e}}}}}{\overline{\gamma_{s m}} \overline{\gamma_{m}}}\left[\pi \rho_{X S} \sqrt{\gamma_{m}\left(1+\gamma_{m}\right)} e^{\frac{1+\gamma_{m}}{\overline{\gamma_{s m}}}} \operatorname{erf}\left(\sqrt{\frac{1+\gamma_{m}}{\overline{\gamma_{s m}}}}\right)\right] d \gamma_{m}$ $=\frac{\pi \rho_{X S} e^{\frac{1}{\gamma_{s m}}}}{\overline{\gamma_{s m}} \overline{\gamma_{m}}} \int_{0}^{\infty} \sqrt{\gamma_{m}\left(1+\gamma_{m}\right)} e^{-\gamma_{m}\left(\frac{1}{\overline{\gamma_{m}}}+\frac{1}{\overline{\gamma_{e}}}-\frac{1}{\overline{\gamma_{s m}}}\right)}$

$\cdot \int_{0}^{\infty} \sqrt{\gamma_{m}\left(1+\gamma_{m}\right)} e^{-\gamma_{m}\left(\frac{1}{\gamma_{m}}+\frac{1}{\gamma_{e}}-\frac{1}{\gamma_{s m}}\right)} \operatorname{erf}\left(\sqrt{\frac{1+\gamma_{m}}{\overline{\gamma_{s m}}}}\right) d \gamma_{m}$

$=\frac{\pi \rho_{X S} e^{\frac{1}{\gamma_{s m}}}}{\overline{\gamma_{s m}} \cdot \overline{\gamma_{m}}} \int_{0}^{\infty} 2 \sqrt{\frac{\gamma_{m}\left(1+\gamma_{m}\right)}{\pi}} \sum_{n=0}^{\infty} \frac{(-1)^{n}\left(\sqrt{\frac{1+\gamma_{m}}{\overline{\gamma_{s m}}}}\right)^{2 n+1}}{n !(2 n+1)}$

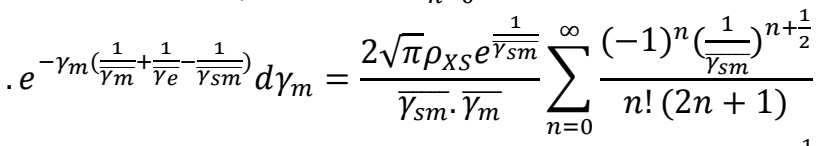

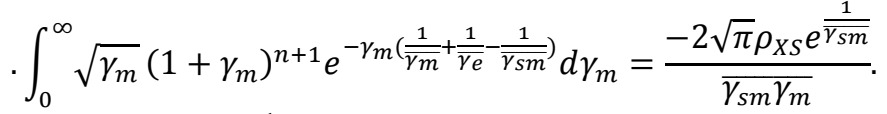

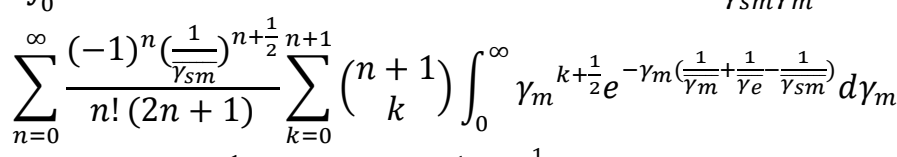

$=-\frac{2 \sqrt{\pi} \rho_{X S} e^{\frac{1}{\overline{\gamma_{s m}}}}}{\overline{\gamma_{s m}} \cdot \overline{\gamma_{m}}} \cdot \sum_{n=0}^{\infty} \frac{(-1)^{n}\left(\frac{1}{\overline{\gamma_{s m}}}\right)^{n+\frac{1}{2}}}{n !(2 n+1)}$

$\sum_{k=0}^{n+1}\left(\begin{array}{c}n+1 \\ k\end{array}\right)\left(\frac{1}{\overline{\gamma_{m}}}+\frac{1}{\overline{\gamma_{e}}}-\frac{1}{\overline{\gamma_{s m}}}\right)^{-k-\frac{3}{2}} \Gamma\left(k+\frac{3}{2}, 1+\frac{\overline{\gamma_{m}}}{\overline{\gamma_{e}}}-\frac{\overline{\gamma_{m}}}{\overline{\gamma_{s m}}}\right)$

$=-\frac{2 \sqrt{\pi} \rho_{X S} e^{\frac{1}{\overline{\gamma_{s m}}}}}{\overline{\gamma_{s m}} \cdot \overline{\gamma_{m}}} \cdot \sum_{n=0}^{\infty} \frac{(-1)^{n}\left(\frac{1}{\overline{\gamma_{s m}}}\right)^{n+\frac{1}{2}}}{n !(2 n+1)}\left(\left(\frac{1}{\overline{\gamma_{m}}}+\frac{1}{\overline{\gamma_{e}}}-\frac{1}{\overline{\gamma_{s m}}}\right)^{-n-\frac{5}{2}}\right.$

$. \Gamma\left(n+\frac{5}{2}, 1+\frac{\overline{\gamma_{m}}}{\overline{\gamma_{e}}}-\frac{\overline{\gamma_{m}}}{\overline{\gamma_{s m}}}\right)+\Gamma(n+2)$

$\left.\sum_{k=0}^{n} \frac{\left(\frac{1}{\overline{\gamma_{m}}}+\frac{1}{\overline{\gamma_{e}}}-\frac{1}{\overline{\gamma_{s m}}}\right)^{-k-\frac{3}{2}} \Gamma\left(k+\frac{3}{2}, 1+\frac{\overline{\gamma_{m}}}{\overline{\gamma_{e}}}-\frac{\overline{\gamma_{m}}}{\overline{\gamma_{s m}}}\right)}{\Gamma(k+1) \Gamma(n+2-k)}\right)=$

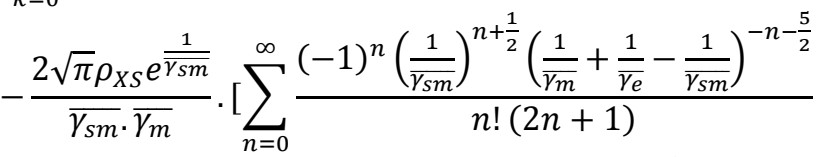

$\Gamma\left(n+\frac{5}{2}, 1+\frac{\overline{\gamma_{m}}}{\overline{\gamma_{e}}}-\frac{\overline{\gamma_{m}}}{\overline{\gamma_{s m}}}\right)+\sum_{n=0}^{\infty} \frac{(-1)^{n}\left(\frac{1}{\overline{\gamma_{s m}}}\right)^{\left(n+\frac{1}{2}\right)} \Gamma(n+2)}{n !(2 n+1)}$

$\left.\left.\sum_{k=0}^{n} \frac{\left(\frac{1}{\overline{\gamma_{m}}}+\frac{1}{\overline{\gamma_{e}}}-\frac{1}{\overline{\gamma_{s m}}}\right)^{-k-\frac{3}{2}} \Gamma\left(k+\frac{3}{2}, 1+\frac{\overline{\gamma_{m}}}{\overline{\gamma_{e}}}-\frac{\overline{\gamma_{m}}}{\overline{\gamma_{s m}}}\right)}{\Gamma(k+1) \Gamma(n+2-k)}\right)\right]$

Similar to (43), the second term of Eq. (42) can be derived as follows:

$C_{2}=$

$\int_{0}^{\infty} \int_{0}^{\gamma_{m}} \frac{e^{-\left(\frac{\gamma_{m}}{\gamma_{m}}+\frac{\gamma_{e}}{\gamma_{e}}\right)}}{\overline{\gamma_{s e}} \overline{\gamma_{e}} \overline{\gamma_{m}}}\left(\int_{0}^{\infty} \log \left(1+\gamma_{e}+\gamma_{s e}+2 \rho_{X s} \sqrt{\gamma_{e} \cdot \gamma_{s e}}\right)\right.$ .$\left.e^{-\frac{\gamma_{s e}}{\bar{\gamma}_{s e}}} d \gamma_{s e}\right) d \gamma_{e} d \gamma_{m}$

$$
\begin{aligned}
C_{2}^{\prime}= & \int_{0}^{\infty} \log \left(1+\gamma_{e}+\gamma_{s e}+2 \rho_{X s} \sqrt{\gamma_{e} \gamma_{s e}}\right) e^{-\frac{\gamma_{s e}}{\gamma_{s e}}} d \gamma_{s e} \\
& =\int_{0}^{\infty} \log \left(1+\gamma_{e}+\gamma_{s e}\right) e^{-\frac{\gamma_{s e}}{\gamma_{s e}}} d \gamma_{s e}+ \\
& \int_{0}^{\infty} \log \left(1+\frac{2 \rho_{X s} \sqrt{\gamma_{e} \gamma_{s e}}}{1+\gamma_{e}+\gamma_{s e}}\right) e^{-\frac{\gamma_{s e}}{\gamma_{s e}}} d \gamma_{s e}=f_{1}{ }^{\prime}+f_{2}{ }^{\prime},
\end{aligned}
$$

Similarly, the logarithmic functions, when $x \ll 1$, can be approximated as (46). So, by exploiting from (33) and (34), we have

$$
\begin{aligned}
f_{1}^{\prime} & =\int_{0}^{\infty} \log \left(1+\gamma_{e}+\gamma_{s e}\right) e^{-\frac{\gamma_{s e}}{\bar{\gamma}_{s e}}} d \gamma_{s e} \\
& =\overline{\gamma_{s e}}\left[\ln \left(1+\gamma_{e}\right)-e^{\frac{1+\gamma_{e}}{\overline{\gamma_{s e}}}} E i\left(-\frac{1+\gamma_{e}}{\overline{\gamma_{s e}}}\right)\right. \\
f_{2}{ }^{\prime} & =\int_{0}^{\infty} \frac{2 \rho_{X s} \sqrt{\gamma_{e} \gamma_{s e}}}{1+\gamma_{e}+\gamma_{s e}} e^{-\frac{\gamma_{s e}}{\gamma_{s e}}} d \gamma_{s e}=2 \rho_{X s} \sqrt{\gamma_{e}\left(1+\gamma_{e}\right)} e^{\frac{1+\gamma_{e}}{\overline{\gamma_{s e}}}} \\
. & \Gamma\left(\frac{3}{2}\right) \Gamma\left(\frac{1}{2}, \frac{1+\gamma_{e}}{\overline{\gamma_{s e}}}\right)=\pi \rho_{X s} \sqrt{\gamma_{e}\left(1+\gamma_{e}\right)} e^{\frac{1+\gamma_{e}}{\gamma_{s e}}} \\
. & \left(1-\operatorname{erf}\left(\sqrt{\frac{1+\gamma_{e}}{\bar{\gamma}_{s e}}}\right)\right),
\end{aligned}
$$

Now, we define $C_{2}{ }^{\prime \prime}$ as

$$
C_{2}^{\prime \prime}=\int_{0}^{\gamma_{m}} e^{-\frac{\gamma e}{\gamma e}}\left(C_{2}^{\prime}\right) d \gamma_{e}
$$

By replacing from (65), we have

$C_{2}^{\prime \prime}=\int_{0}^{\gamma_{m}} e^{-\frac{\gamma_{e}}{\gamma_{e}}}\left[\overline{\gamma_{s e}} \ln \left(1+\gamma_{e}\right)-\overline{\gamma_{s e}} e^{\frac{1+\gamma_{e}}{\overline{\gamma_{s e}}}} E i\left(-\frac{1+\gamma_{e}}{\overline{\gamma_{s e}}}\right)+\right.$ $\pi \rho_{X S} \sqrt{\gamma_{e}\left(1+\gamma_{e}\right)} e^{\frac{1+\gamma_{e}}{\overline{\gamma_{s e}}}}-\pi \rho_{X S} \sqrt{\gamma_{e}\left(1+\gamma_{e}\right)} e^{\frac{1+\gamma_{e}}{\overline{\gamma_{s e}}}}$ . $\left.\operatorname{erf}\left(\sqrt{\frac{1+\gamma_{e}}{\overline{\gamma_{s e}}}}\right)\right] d \gamma_{e}=i_{1}+i_{2}+i_{3}+i_{4}$,

where, by exploiting from (29-35), we have

$$
\begin{aligned}
i_{1}= & \int_{0}^{\gamma_{m}} \overline{\gamma_{s e}} e^{-\frac{\gamma_{e}}{\bar{\gamma}}} \ln \left(1+\gamma_{e}\right) d \gamma_{e}=\overline{\gamma_{s e}} \overline{\gamma_{e}}\left[e^{\frac{1}{\overline{\gamma e}}} E i\left(-\frac{1+\gamma_{m}}{\overline{\gamma_{e}}}\right)-\right. \\
& \left.e^{-\frac{\gamma_{m}}{\bar{\gamma}_{e}}} \ln \left(1+\gamma_{m}\right)-e^{\frac{1}{\overline{\gamma_{e}}}} E i\left(\frac{-1}{\overline{\gamma_{e}}}\right)\right],
\end{aligned}
$$

and

$$
\begin{aligned}
& i_{2}=-\int_{0}^{\gamma_{m}} \overline{\gamma_{s e}} e^{-\frac{\gamma_{e}}{\gamma_{e}}} e^{\frac{1+\gamma_{e}}{\overline{\gamma_{s e}}}} E i\left(-\frac{1+\gamma_{e}}{\overline{\gamma_{s e}}}\right) d \gamma_{e}= \\
& -\overline{\gamma_{s e}}\left(\frac{\overline{\gamma_{s e}} \cdot \overline{\gamma_{e}}}{\overline{\gamma_{s e}}-\overline{\gamma_{e}}}\right) e^{\frac{1}{\bar{\gamma}_{s e}}}\left[e^{\frac{1}{\overline{\gamma_{e}}}-\frac{1}{\bar{\gamma}_{s e}}} E i\left(-\frac{1+\overline{\gamma_{m}}}{\overline{\gamma_{s e}}}\right)-e^{\gamma_{m}\left(\frac{1}{\bar{\gamma}_{e}}-\frac{1}{\bar{\gamma}_{s e}}\right.}\right) \\
& \left.. E i\left(-\frac{1+\overline{\gamma_{m}}}{\overline{\gamma_{s e}}}\right)-e^{\frac{1}{\overline{\gamma_{e}}}-\frac{1}{\bar{\gamma}_{s e}}} E i\left(-\frac{1}{\overline{\gamma_{e}}}\right)+E i\left(-\frac{1}{\overline{\gamma_{s e}}}\right)\right] . \\
& i_{3}=\int_{0}^{\gamma_{m}} e^{-\frac{\gamma_{e}}{\bar{\gamma}_{e}}}\left[\pi \rho_{X S} \sqrt{\gamma_{e}\left(1+\gamma_{e}\right)} e^{\frac{1+\gamma_{e}}{\bar{\gamma}_{s e}}}\right] d \gamma_{e} \\
& =\pi \rho_{X S} e^{\frac{1}{\bar{\gamma}_{s e}}} \int_{0}^{\gamma_{m}} \sqrt{\gamma_{e}\left(1+\gamma_{e}\right)} e^{-\gamma_{e}\left(\frac{1}{\gamma_{e}}-\frac{1}{\gamma_{s e}}\right)} d \gamma_{e}
\end{aligned}
$$

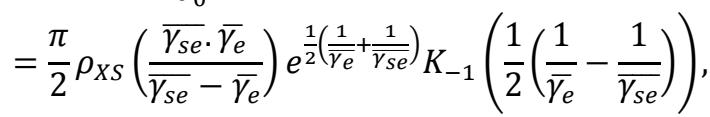

$$
\begin{aligned}
& i_{4}=-\int_{0}^{\gamma_{m}} e^{-\frac{\gamma_{e}}{\bar{\gamma}}}\left[\pi \rho_{X s} \sqrt{\gamma_{e}\left(1+\gamma_{e}\right)} e^{\frac{1+\gamma_{e}}{\overline{\gamma_{s e}}}} \cdot \operatorname{erf}\left(\sqrt{\frac{1+\gamma_{e}}{\overline{\gamma_{s e}}}}\right)\right]
\end{aligned}
$$


$. d \gamma_{e}=-\pi \rho_{X S} e^{\frac{1}{\overline{\gamma_{s e}}}} \int_{0}^{\gamma_{m}} \sqrt{\gamma_{e}\left(1+\gamma_{e}\right)} e^{-\gamma_{e}\left(\frac{1}{\overline{\gamma e}}-\frac{1}{\gamma_{s e}}\right)}$

. $\operatorname{erf}\left(\sqrt{\frac{1+\gamma_{e}}{\overline{\gamma_{s e}}}}\right) d \gamma_{e}$

Thus

$C_{2}=\int_{0}^{\infty} \frac{e^{-\frac{\gamma_{m}}{\gamma_{m}}}}{\overline{\gamma_{s e}} \cdot \overline{\gamma_{e}} \cdot \overline{\gamma_{m}}}\left(i_{1}+i_{2}+i_{3}+i_{4}\right) d \gamma_{m}=\sum_{i=1}^{9} t_{i}$,

where, by exploiting (29-35), we have

$t_{1}=\int_{0}^{\infty} \frac{e^{-\frac{\gamma_{m}}{\overline{\gamma m}}} e^{\frac{1}{\bar{\gamma}}}}{\overline{\gamma_{m}}} \operatorname{Ei}\left(-\frac{1+\gamma_{m}}{\overline{\gamma_{e}}}\right) d \gamma_{m}=$

$e^{\frac{1}{\overline{\gamma e}}}\left[E i\left(-\frac{1}{\overline{\gamma_{e}}}\right)-e^{\frac{1}{\overline{\gamma m}}} \cdot E i\left(-\left(\frac{1}{\overline{\gamma_{m}}}+\frac{1}{\overline{\gamma_{e}}}\right)\right)\right]$,

$t_{2}=\int_{0}^{\infty} \frac{e^{-\frac{\gamma_{m}}{\gamma_{m}}} e^{-\frac{\gamma_{m}}{\gamma_{e}}}}{\overline{\gamma_{m}}} \ln \left(1+\gamma_{m}\right) d \gamma_{m}$

$=-\frac{\overline{\gamma_{e}}}{\overline{\gamma_{m}}+\overline{\gamma_{e}}} e^{\frac{1}{\bar{\gamma}}+\frac{1}{\overline{\gamma_{e}}}} E i\left(-\left(\frac{1}{\overline{\gamma_{m}}}+\frac{1}{\overline{\gamma_{e}}}\right)\right)$,

$t_{3}=-\int_{0}^{\infty} \frac{e^{-\frac{\gamma_{m}}{\overline{\gamma_{m}}}} e^{\frac{1}{\bar{\gamma}}}}{\overline{\overline{\gamma_{m}}}} E i\left(-\frac{1}{\overline{\gamma_{e}}}\right) d \gamma_{m}=-e^{\frac{1}{\overline{\gamma_{e}}}} E i\left(-\frac{1}{\overline{\gamma_{e}}}\right)$,

$t_{4}=-\int_{0}^{\infty} \frac{e^{-\frac{\gamma_{m}}{\bar{\gamma}_{m}}} e^{\frac{1}{\bar{\gamma}_{e}}}}{\overline{\overline{\gamma_{m}}}} \frac{\overline{\gamma_{s e}}}{\overline{\gamma_{s e}}-\overline{\gamma_{e}}} E i\left(-\frac{1+\overline{\gamma_{m}}}{\overline{\gamma_{s e}}}\right) d \gamma_{m}$

$=-\frac{\overline{\gamma_{s e}}}{\overline{\gamma_{s e}}-\overline{\overline{\gamma_{e}}}} e^{\frac{1}{\overline{\gamma e}}}\left[E i\left(-\frac{1}{\overline{\gamma_{s e}}}\right)-e^{\frac{1}{\overline{\gamma m}}} E i\left(-\left(\frac{1}{\overline{\gamma_{m}}}+\frac{1}{\overline{\gamma_{s e}}}\right)\right)\right]$,

$t_{5}=\int_{0}^{\infty} \frac{e^{-\frac{\gamma_{m}}{\overline{\gamma_{m}}}} e^{\frac{1}{\bar{\gamma}_{s e}}} e^{\gamma_{m}\left(\frac{1}{\overline{\gamma_{e}}}-\frac{1}{\overline{\gamma_{s e}}}\right)}}{\overline{\gamma_{m}}} \frac{\overline{\gamma_{s e}}}{\overline{\gamma_{s e}}-\overline{\gamma_{e}}} E i\left(-\frac{1+\overline{\gamma_{m}}}{\overline{\gamma_{s e}}}\right) d \gamma_{m}$

$=\frac{e^{\frac{1}{\gamma_{s e}}}}{\overline{\gamma_{m}}} \cdot \frac{\overline{\gamma_{s e}}}{\overline{\gamma_{s e}}-\overline{\gamma_{e}}} \cdot \frac{1}{\frac{1}{\overline{\gamma_{m}}}-\frac{1}{\overline{\gamma_{e}}}+\frac{1}{\overline{\gamma_{s e}}}} \cdot\left(E i\left(-\frac{1}{\overline{\gamma_{s e}}}\right)-\right.$

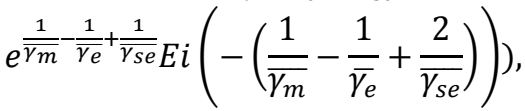

$t_{6}=\int_{0}^{\infty} \frac{e^{-\frac{\gamma_{m}}{\overline{\gamma_{m}}}} \frac{1}{\overline{\gamma_{e}}}}{\overline{\gamma_{m}}} \cdot \frac{\overline{\gamma_{s e}}}{\overline{\gamma_{s e}}-\overline{\gamma_{e}}} E i\left(-\frac{1}{\overline{\gamma_{s e}}}\right) d \gamma_{m}$

$=\frac{\overline{\gamma_{s e}}}{\overline{\gamma_{s e}}-\overline{\gamma_{e}}} e^{\frac{1}{\overline{\gamma_{e}}}} E i\left(-\frac{1}{\overline{\gamma_{s e}}}\right)$,

$t_{7}=-\int_{0}^{\infty} \frac{e^{-\frac{\gamma_{m}}{\overline{\gamma_{m}}}} e^{\frac{1}{\overline{\gamma_{s e}}}}}{\overline{\gamma_{m}}} \cdot \frac{\overline{\gamma_{s e}}}{\overline{\gamma_{s e}}-\overline{\gamma_{e}}} E i\left(-\frac{1}{\overline{\gamma_{s e}}}\right) d \gamma_{m}$

$=-\frac{\overline{\gamma_{s e}}}{\overline{\gamma_{s e}}-\overline{\gamma_{e}}} e^{\frac{1}{\overline{\gamma_{s e}}}} E i\left(-\frac{1}{\overline{\gamma_{s e}}}\right)$,

$t_{8}=\int_{0}^{\infty} \frac{\frac{\pi}{2} \rho_{X S} e^{\frac{1}{2}\left(\frac{1}{\overline{\gamma_{e}}}+\frac{1}{\overline{\gamma_{s e}}}\right)}}{\overline{\overline{\gamma_{m}}}\left(\overline{\overline{\gamma_{s e}}}-\overline{\overline{\gamma_{e}}}\right)} K_{-1}\left(\frac{1}{2}\left(\frac{1}{\overline{\gamma_{e}}}-\frac{1}{\overline{\gamma_{s e}}}\right)\right) e^{-\frac{\gamma_{m}}{\overline{\gamma_{m}}}} d \gamma_{m}$

$=\frac{\pi}{2} \cdot \frac{\rho_{X s} e^{\frac{1}{2}\left(\frac{1}{\bar{\gamma}}+\frac{1}{\overline{\gamma_{s e}}}\right)}}{\left(\overline{\gamma_{s e}}-\overline{\gamma_{e}}\right)} K_{-1}\left(\frac{1}{2}\left(\frac{1}{\overline{\gamma_{e}}}-\frac{1}{\overline{\gamma_{s e}}}\right)\right)$,

$t_{9}=-\frac{\pi \rho_{X s} e^{\frac{1}{\overline{\gamma_{s e}}}}}{\overline{\gamma_{s e}} \cdot \overline{\gamma_{e}} \cdot \overline{\gamma_{m}}} \int_{0}^{\infty} e^{-\frac{\gamma_{m}}{\overline{\gamma_{m}}}}\left(\int_{0}^{\gamma_{m}} \sqrt{\gamma_{e}\left(1+\gamma_{e}\right)} e^{-\gamma_{e}\left(\frac{1}{\bar{\gamma}_{e}}-\frac{1}{\bar{\gamma}_{s e}}\right)}\right.$
. $\left.\operatorname{erf}\left(\sqrt{\frac{1+\gamma_{e}}{\overline{\gamma_{s e}}}}\right) d \gamma_{e}\right) d \gamma_{m}=\frac{-\pi \rho_{X s} e^{\frac{1}{\gamma_{s e}}}}{\overline{\gamma_{s e}} \overline{\gamma_{e}} \overline{\gamma_{m}}} \int_{0}^{\gamma_{m}}($

$\left.\sqrt{\gamma_{e}\left(1+\gamma_{e}\right)} e^{-\gamma_{e}\left(\frac{1}{\overline{\gamma_{e}}}-\frac{1}{\overline{\gamma_{s e}}}\right)} \operatorname{erf}\left(\sqrt{\frac{1+\gamma_{e}}{\overline{\gamma_{s e}}}}\right)\left(\int_{\gamma_{e}}^{\infty} e^{-\frac{\gamma_{m}}{\overline{\gamma_{m}}}} d \gamma_{m}\right)\right)$

$. d \gamma_{e}=-\frac{\pi \rho_{X S} e^{\frac{1}{\overline{\gamma_{s e}}}} \int_{0}^{\gamma_{m}} \sqrt{\gamma_{e}\left(1+\gamma_{e}\right)} e^{-\gamma_{e}\left(\frac{1}{\overline{\gamma_{e}}}+\frac{1}{\overline{\gamma_{m}}}-\frac{1}{\overline{\gamma_{s e}}}\right)}}{\overline{\overline{\gamma_{s e}}} \cdot \overline{\gamma_{e}}}$

.erf $\left(\sqrt{\frac{1+\gamma_{e}}{\overline{\gamma_{s e}}}}\right) d \gamma_{e}=-\frac{\pi \rho_{X s} e^{\frac{1}{\bar{\gamma}_{s e}}}}{\overline{\gamma_{s e}} \cdot \overline{\gamma_{e}}} \int_{0}^{\gamma_{m}} 2 \sqrt{\frac{\gamma_{e}\left(1+\gamma_{e}\right)}{\pi}}$

$\cdot \sum_{n=0}^{\infty} \frac{(-1)^{n}\left(\sqrt{\frac{1+\gamma_{e}}{\overline{\gamma_{s e}}}}\right)^{2 n+1}}{n !(2 n+1)} e^{-\gamma_{e}\left(\frac{1}{\overline{\gamma_{e}}}+\frac{1}{\gamma_{m}}-\frac{1}{\bar{\gamma}_{s e}}\right)} d \gamma_{e}=-\frac{2 \sqrt{\pi} \rho_{X S} e^{\frac{1}{\overline{\gamma_{s e}}}}}{\overline{\gamma_{s e}} \cdot \overline{\gamma_{e}}}$

$\cdot \sum_{n=0}^{\infty} \frac{(-1)^{n}\left(\frac{1}{\bar{\gamma}_{s e}}\right)^{n+\frac{1}{2}}}{n !(2 n+1)} \cdot \int_{0}^{\gamma_{m}} \sqrt{\gamma_{e}}\left(1+\gamma_{e}\right)^{n+1} e^{-\gamma_{e}\left(\frac{1}{\overline{\gamma_{e}}}+\frac{1}{\overline{\gamma_{m}}}-\frac{1}{\overline{\gamma_{s e}}}\right)} d \gamma_{e}$

$=-\frac{2 \sqrt{\pi} \rho_{X s} e^{\frac{1}{\gamma_{s e}}}}{\overline{\gamma_{s e}} \cdot \overline{\gamma_{e}}} \sum_{n=0}^{\infty} \frac{(-1)^{n}\left(\frac{1}{\overline{\gamma_{s e}}}\right)^{n+\frac{1}{2}}}{n !(2 n+1)} \cdot \sum_{k=0}^{n+1}\left(\begin{array}{c}n+1 \\ k\end{array}\right)$

$\int_{0}^{\gamma_{m}} \gamma_{e}^{k+\frac{1}{2}} \cdot e^{-\gamma_{e}\left(\frac{1}{\overline{\gamma_{e}}+\frac{1}{\gamma_{m}}-\frac{1}{\gamma_{s e}}}\right)} d \gamma_{e}=\frac{2 \sqrt{\pi} \rho_{X s} e^{\frac{1}{\overline{\gamma_{s e}}}}}{\overline{\gamma_{s e}} \cdot \overline{\gamma_{e}}}$

$\sum_{n=0}^{\infty} \frac{(-1)^{n}\left(\frac{1}{\overline{\gamma_{s e}}}\right)^{n+\frac{1}{2}}}{n !(2 n+1)} \cdot \sum_{k=0}^{n+1}\left(\begin{array}{c}n+1 \\ k\end{array}\right)\left(\frac{1}{\overline{\gamma_{e}}}+\frac{1}{\overline{\gamma_{m}}}-\frac{1}{\overline{\gamma_{s e}}}\right)^{-k-\frac{3}{2}}$

$.\left(\Gamma\left(k+\frac{3}{2},\left(1+\frac{\overline{\gamma_{m}}}{\overline{\gamma_{e}}}-\frac{\overline{\gamma_{m}}}{\overline{\gamma_{s e}}}\right)\right)-\Gamma\left(k+\frac{3}{2}, 0\right)\right)=\frac{2 \sqrt{\pi} \rho_{X S}}{\overline{\gamma_{s e}} \overline{\gamma_{e}}}$

$\cdot e^{\frac{1}{\bar{\gamma}_{s e}}} \sum_{n=0}^{\infty} \frac{(-1)^{n}\left(\frac{1}{\bar{\gamma}_{s e}}\right)^{n+\frac{1}{2}}}{n !(2 n+1)} \cdot\left[\sum_{k=0}^{n}\left(\begin{array}{c}n+1 \\ k\end{array}\right) \frac{\left(\frac{1}{\overline{\gamma_{e}}}+\frac{1}{\overline{\gamma_{m}}}-\frac{1}{\overline{\gamma_{s e}}}\right)^{-k}}{\sqrt{\left(\frac{1}{\overline{\gamma_{e}}}+\frac{1}{\overline{\gamma_{m}}}-\frac{1}{\overline{\gamma_{s e}}}\right)^{3}}}\right.$

$.\left(\Gamma\left(k+\frac{3}{2},\left(1+\frac{\overline{\gamma_{m}}}{\overline{\gamma_{e}}}-\frac{\overline{\gamma_{m}}}{\overline{\gamma_{s e}}}\right)\right)-\Gamma\left(k+\frac{3}{2}, 0\right)\right)+$

$\frac{\left(\frac{1}{\overline{\gamma_{e}}}+\frac{1}{\overline{\gamma_{m}}}-\frac{1}{\bar{\gamma}_{s e}}\right)^{-n}}{\sqrt{\left(\frac{1}{\overline{\gamma_{e}}}+\frac{1}{\bar{\gamma}_{m}}-\frac{1}{\overline{\gamma_{s e}}}\right)^{5}}}\left(\Gamma\left(n+\frac{5}{2},\left(1+\frac{\overline{\gamma_{m}}}{\overline{\gamma_{e}}}-\frac{\overline{\gamma_{m}}}{\overline{\gamma_{s e}}}\right)\right)-\right.$

$\left.\left.\Gamma\left(n+\frac{5}{2}, 0\right)\right)\right]=\frac{2 \sqrt{\pi} \rho_{X S} e^{\frac{1}{\overline{s e}_{s e}}}}{\overline{\gamma_{s e}} \cdot \overline{\gamma_{e}}}\left[\sum_{n=0}^{\infty} \frac{(-1)^{n}\left(\frac{1}{\overline{\gamma_{s e}}}\right)^{n+\frac{1}{2}}}{n !(2 n+1)}\right.$

$\left(\frac{1}{\overline{\gamma_{e}}}+\frac{1}{\overline{\gamma_{m}}}-\frac{1}{\overline{\gamma_{s e}}}\right)^{-n-\frac{5}{2}}\left(\Gamma\left(n+\frac{5}{2},\left(1+\frac{\overline{\gamma_{m}}}{\overline{\gamma_{e}}}-\frac{\overline{\gamma_{m}}}{\overline{\gamma_{s e}}}\right)\right)-\right.$

$\left.\Gamma\left(n+\frac{5}{2}, 0\right)\right)+\sum_{n=0}^{\infty} \frac{(-1)^{n}\left(\frac{1}{\overline{\gamma_{s e}}}\right)^{n+\frac{1}{2}}}{n !(2 n+1)} \sum_{k=0}^{n}\left(\begin{array}{c}n+1 \\ k\end{array}\right)$

$\cdot\left(\frac{1}{\overline{\gamma_{e}}}+\frac{1}{\overline{\gamma_{m}}}-\frac{1}{\overline{\gamma_{s e}}}\right)^{-k-\frac{3}{2}} \cdot\left(\Gamma\left(k+\frac{3}{2},\left(1+\frac{\overline{\gamma_{m}}}{\overline{\gamma_{e}}}-\frac{\overline{\gamma_{m}}}{\overline{\gamma_{s e}}}\right)\right)-\right.$

$\left.\left.\Gamma\left(k+\frac{3}{2}, 0\right)\right)\right]$. 
Therefore, from (42), by computing $C_{s}^{a v g}=\sum_{q=1}^{8} k_{q}-\sum_{q=1}^{9} t_{q}$ and considering the obtained approximation in (40), the proof is completed.

\section{Appendix C:}

Proof of Theorem 3: By exploiting of (13-Corollary 1) and (17), we have

$P_{C}=P\left(\log \left(1+\left(1-\rho_{X S}^{2}\right) \gamma_{m}\right)>R_{S}\right)=P\left(\gamma_{m}>\frac{2^{R_{S}}-1}{1-\rho_{X S}^{2}}\right)=$

$\int_{0}^{\infty} \int_{\frac{{ }_{2} R_{S-1}}{1-\rho_{X S}^{2}}}^{\infty} f\left(\gamma_{m}, \gamma_{e}\right) d \gamma_{m} \cdot d \gamma_{e}=\mathrm{e}^{\frac{1-2^{2} R_{S}}{\left(1-\rho_{X S}^{2}\right) \overline{\gamma_{m}}}}$,

where by using (16), the asymptotic SOP is given as follows:

$$
P_{S O P}=1-\mathrm{e}^{\frac{1-2^{R}}{\left(1-\rho_{X S}^{2}\right) \overline{\gamma m}}}
$$

Also, for Corollary 2, by exploiting of (13-Corollary 2) and (17), we have

$P_{C}=P\left(\log \left(\frac{1+\gamma_{m}+\gamma_{s m}+2 \rho_{X s} \sqrt{\gamma_{m} \gamma_{s m}}}{1+\gamma_{e}+\gamma_{s e}+2 \rho_{X s} \sqrt{\gamma_{e} \gamma_{s e}}}\right)>R_{s}\right)=$

$P\left(\gamma_{m}+2 \rho_{X s} \sqrt{\gamma_{m} \gamma_{s m}}>2^{R_{s}}\left(1+\gamma_{e}+\gamma_{s e}+2 \rho_{X s} \sqrt{\gamma_{e} \gamma_{s e}}\right)\right.$

$-\left(1+\gamma_{s m}\right)=P\left(\sqrt{\gamma_{m}}>\frac{1}{2} \sqrt{w}-\rho_{X s} \sqrt{\gamma_{s m}}\right)<P\left(\sqrt{\gamma_{m}}>\right.$

$\left.\frac{1}{2} \sqrt{w}\right)=P\left(\gamma_{m}>\frac{1}{4} w\right)=P_{C}^{U P}$,

Where $\quad w=4\left[2^{R_{s}}\left(1+\gamma_{e}+\gamma_{s e}+2 \rho_{X s} \sqrt{\gamma_{e} \gamma_{s e}}\right)-\gamma_{s m}(1-\right.$ $\left.\left.\rho_{X S}^{2}\right)-1\right]$. By exploiting from (36-38), we have

$P_{C}^{U P}=\int_{0}^{\infty} \int_{0}^{\infty} \int_{0}^{\infty} \int_{\frac{w}{4}}^{\infty} f\left(\gamma_{m}, \gamma_{s m}, \gamma_{s e}, \gamma_{e}\right) d \gamma_{m} d \gamma_{e} d \gamma_{s e} d \gamma_{s m}=$ $\int_{0}^{\infty} \int_{0}^{\infty} \int_{0}^{\infty} \int_{\underline{w}}^{\infty} \frac{1}{\overline{\gamma_{s e} \gamma_{s m}} \overline{\gamma_{e}} \overline{\gamma_{m}}} e^{-\frac{\gamma_{m}}{\gamma_{m}}} e^{-\frac{\gamma_{s e}}{\gamma_{s e}}} e^{-\frac{\gamma_{s m}}{\gamma_{s m}}} e^{-\frac{\gamma_{e}}{\overline{\gamma_{e}}}} d \gamma_{m} d \gamma_{e} d \gamma_{s e} d \gamma_{s m}$ $=\int_{0}^{\infty} \int_{0}^{\infty} \int_{0}^{\infty} \frac{1}{\overline{\gamma_{s e} \gamma_{s m}} \overline{\gamma_{e}} \overline{\gamma_{m}}} e^{-\frac{\gamma_{s e}}{\bar{\gamma}_{s e}}} e^{-\frac{\gamma_{s m}}{\bar{\gamma}_{s m}}} e^{-\frac{\gamma_{e}}{\bar{\gamma}_{e}}}\left(\int_{\frac{w}{4}}^{\infty} e^{-\frac{\gamma_{m}}{\overline{\gamma_{m}}}} d \gamma_{m}\right) d \gamma_{e}$ $d \gamma_{s e} d \gamma_{s m}=\int_{0}^{\infty} \int_{0}^{\infty} \frac{e^{-\frac{\gamma_{s e}}{\bar{\gamma}_{s e}}} e^{-\frac{\gamma_{s m}}{\bar{\gamma}_{s m}}}}{\overline{\gamma_{s e} \gamma_{s m}} \overline{\gamma_{e}}} e^{\frac{1}{\overline{\gamma_{m}}}\left(2^{R_{s}}\left(1+\gamma_{s e}\right)-\gamma_{s m}\left(1-\rho_{X S}^{2}\right)-1\right)}$ $\cdot\left(\int_{0}^{\infty} e^{-\gamma_{e}\left(\frac{1}{\overline{\gamma e}}+\frac{2^{R}}{\overline{\gamma_{m}}}\right)} \cdot e^{-\sqrt{\gamma_{e}}\left(2^{R_{S}+1} \rho_{X S} \sqrt{\gamma_{s e}}\right) \frac{1}{\overline{\gamma_{m}}}} d \gamma_{e}\right) d \gamma_{s e} d \gamma_{s m}$ $=\int_{0}^{\infty} \int_{0}^{\infty} \frac{1}{\overline{\gamma_{s e} \gamma_{s m}}} \overline{\overline{\gamma_{e}}} e^{-\frac{\gamma_{s e}}{\gamma_{s e}}} e^{-\frac{\gamma_{s m}}{\gamma_{s m}}} e^{\frac{1}{\overline{\gamma m}}\left(2^{R}\left(1+\gamma_{s e}\right)-\gamma_{s m}\left(1-\rho_{X S}^{2}\right)-1\right)}$ $\left(\frac{\overline{\gamma_{e}} \overline{\gamma_{m}}}{\overline{\gamma_{m}}+\overline{\gamma_{e}} 2^{R_{s}}}-\frac{\sqrt{\pi}\left(2^{R_{s}} \rho_{X S} \sqrt{\gamma_{s e}}\right)}{\overline{\gamma_{m}}\left(\frac{1}{\overline{\gamma_{e}}}+\frac{2^{R_{s}}}{\overline{\gamma_{m}}}\right)^{\frac{3}{2}}}\left(1-\operatorname{erf}\left(\frac{2^{R_{s}} \rho_{X S} \sqrt{\gamma_{s e}}}{\overline{\overline{\gamma_{m}}} \sqrt{\frac{1}{\overline{\gamma_{e}}}+\frac{2^{R_{s}}}{\overline{\gamma_{m}}}}}\right)\right)\right.$ $e^{\frac{2^{2 R_{S}} \rho_{X S}^{2} \gamma_{s e}}{\overline{\gamma m}^{2}\left(\frac{1}{\gamma_{e}}+\frac{2^{R} \bar{\gamma}}{\gamma_{m}}\right)}} d \gamma_{s e} d \gamma_{s m}=V_{1}-V_{2}-V_{3}$

where

$V_{1}=\frac{\overline{\gamma_{m}} e^{\frac{1}{\overline{\gamma m}}\left(2^{R_{S}-1}\right)}}{\left(\overline{\gamma_{m}}+\overline{\gamma_{e}} 2^{R_{S}}\right)\left(1-\frac{\overline{\gamma_{s e}} 2^{R_{S}}}{\overline{\gamma_{m}}}+\frac{\overline{\gamma_{s m}}\left(1-\rho_{X S}^{2}\right)}{\overline{\gamma_{m}}}-\frac{\overline{\gamma_{s e} \gamma^{2}} 2^{2 R_{S}} \rho_{X S}^{2}}{\overline{\gamma_{m}}\left(\overline{\gamma_{m}}+\overline{\gamma_{e}} 2^{R_{s}}\right)}\right)}$

$$
=\frac{\pi\left(2^{R_{S}-1} \rho_{X S}\right) e^{\frac{\left(2^{R_{S}-1}\right)}{\overline{\gamma_{m}}}}}{\overline{\gamma_{m}} \overline{\gamma_{e}}\left(\frac{1}{\overline{\gamma_{e}}}+\frac{2^{R_{S}}}{\overline{\gamma_{m}}}\right)^{\frac{3}{2}}\left(1-\frac{\overline{\gamma_{s e}} 2^{R_{S}}}{\overline{\gamma_{m}}}+\frac{\overline{\gamma_{S m}}\left(1-\rho_{X S}^{2}\right)}{\overline{\gamma_{m}}}-\frac{\overline{\gamma_{S e} \gamma_{e}} 2^{2 R_{S}} \rho_{X S}^{2}}{\overline{\gamma_{m}}\left(\overline{\gamma_{m}}+\overline{\gamma_{e}} 2^{R_{S}}\right)}\right)^{\frac{3}{2}}},
$$$$
V_{3}=\frac{\sqrt{\pi}\left(2^{R_{S}} \rho_{X S}\right) e^{\frac{\left(2^{R_{S-1}}\right)}{\overline{\gamma_{m}}}}}{\overline{\gamma_{m}} \overline{\gamma_{e}}\left(\frac{1}{\overline{\gamma_{e}}}+\frac{2^{R_{S}}}{\overline{\gamma_{m}}}\right)^{\frac{3}{2}}} \cdot\left[\frac{\sqrt{\pi}}{2}-\frac{1}{\sqrt{\pi}} \tan ^{-1}\left(\frac{\overline{\gamma_{m}}}{2^{R_{S}} \rho_{X S}}\right.\right.
$$

$\cdot \sqrt{\left.\left(\frac{1}{\overline{\gamma_{e}}}+\frac{2^{R_{S}}}{\overline{\gamma_{m}}}\right)\left(\frac{1}{\overline{\gamma_{s e}}}-\frac{\overline{\gamma_{s e}} 2^{R_{S}}}{\overline{\gamma_{m}}}+\frac{\overline{\gamma_{s m}}\left(1-\rho_{X S}^{2}\right)}{\overline{\gamma_{m}}}-\frac{\overline{\gamma_{s e}} \overline{\gamma_{e}} 2^{2 R_{s}} \rho_{X S}^{2}}{\overline{\gamma_{m}}\left(\overline{\gamma_{m}}+\overline{\gamma_{e}} 2^{R_{S}}\right)}\right)\right]}$

$\cdot \frac{1}{\left(1-\frac{\overline{\gamma_{s e}} 2^{R_{S}}}{\overline{\gamma_{m}}}+\frac{\overline{\gamma_{s m}}\left(1-\rho_{X S}^{2}\right)}{\overline{\gamma_{m}}}-\frac{\overline{\gamma_{e}} 2^{2 R_{S}} \rho_{X S}^{2}}{\overline{\gamma_{m}}\left(\overline{\gamma_{m}}+\overline{\gamma_{e}} 2^{R_{S}}\right)}\right)^{\frac{3}{2}}}+\left(\frac{\frac{1}{\sqrt{\pi}} 2^{R_{S}} \rho_{X S}}{\overline{\frac{\gamma_{m}}{\sqrt{\gamma_{e}}}+\frac{2^{R_{S}}}{\overline{\gamma_{m}}}}}\right)$

$\overline{\left(1-\frac{\overline{\gamma_{s e}} 2^{R_{S}}}{\overline{\gamma_{m}}}+\frac{\overline{\gamma_{s m}}\left(1-\rho_{X S}^{2}\right)}{\overline{\gamma_{m}}}-\frac{\overline{\gamma_{s e} \gamma_{e}} 2^{2 R_{S}} \rho_{X S}^{2}}{\overline{\gamma_{m}}\left(\overline{\gamma_{m}}+\overline{\gamma_{e}} 2^{R_{S}}\right)}\right)\left(1-\frac{\overline{\gamma_{s e}} 2^{R_{S}}}{\overline{\gamma_{m}}}+\frac{\overline{\gamma_{s m}}\left(1-\rho_{X S}^{2}\right)}{\overline{\gamma_{m}}}\right)}$.

So, by using (16), the upper bound for asymptotic SOP is given as (19). So, the proof is completed.

Funding. This research is supported by the Faculty of Engineering at the Ferdowsi University of Iran.

\section{Compliance with ethical standards}

Conflict of interest. The authors declare that they have no m conflict of interest.

Ethical approval. This article does not contain any studies with human participants or animals performed by any of the authors.

Availability of data. Not applicable.

Code availability. Not applicable.

\section{References}

[1] Schneier, Bruce. "Cryptographic design vulnerabilities." Computer 31, no. 9 (1998): 29-33.

[2] Shannon, Claude E. "Communication theory of secrecy systems." The Bell system technical journal 28, no. 4 (1949): 656-715.

[3] Wyner, Aaron D. "The wire-tap channel." Bell system technical journal 54, no. 8 (1975): 1355-1387.

[4] Csiszár, Imre, and Janos Korner. "Broadcast channels with confidential messages." IEEE transactions on information theory 24, no. 3 (1978): 339-348.

[5] Leung-Yan-Cheong, S., and M. Hellman. "The Gaussian wire-tap channel." IEEE transactions on information theory 24, no. 4 (1978): 451456.

[6] Bloch, Matthieu, João Barros, Miguel RD Rodrigues, and Steven W. McLaughlin. "Wireless information-theoretic security." IEEE Transactions on Information Theory 54, no. 6 (2008): 2515-2534.

[7] Ai, Yun, Long Kong, and Michael Cheffena. "Secrecy outage analysis of double shadowed Rician channels." Electronics Letters 55, no. 13 (2019): 765-767. 
[8] Li, Jiangyuan, and Athina P. Petropulu. "Ergodic secrecy rate for multiple-antenna wiretap channels with Rician fading." IEEE Transactions on Information Forensics and Security 6, no. 3 (2011): 861867.

[9] Liu, Xian. "Probability of strictly positive secrecy capacity of the RicianRician fading channel." IEEE Wireless Communications Letters 2, no. 1 (2012): 50-53.

[10] Omri, Aymen, and Mazen O. Hasna. "Average secrecy outage rate and average secrecy outage duration of wireless communication systems with diversity over Nakagami-m fading channels." IEEE Transactions on Wireless Communications 17, no. 6 (2018): 3822-3833.

[11] Lei, Hongjiang, Chao Gao, Yongcai Guo, and Gaofeng Pan. "On physical layer security over generalized Gamma fading channels." IEEE Communications Letters 19, no. 7 (2015): 1257-1260.

[12] Lei, Hongjiang, Imran Shafique Ansari, Gaofeng Pan, Basel Alomair, and Mohamed-Slim Alouini. "Secrecy capacity analysis over \$ $\backslash$ alpha-Imu \$ fading channels." IEEE Communications Letters 21, no. 6 (2017): 14451448 .

[13] Moualeu, Jules M., Daniel Benevides da Costa, F. Javier Lopez-Martinez, Walaa Hamouda, Telex MN Nkouatchah, and Ugo Silva Dias. "Transmit Antenna Selection in Secure MIMO Systems Over Fading Channels." IEEE Transactions on Communications 67, no. 9 (2019): 6483-6498.

[14] Kong, Long, and Georges Kaddoum. "On Physical Layer Security Over the Fisher-Snedecor Wiretap Fading Channels." IEEE Access 6 (2018): 39466-39472.

[15] Badarneh, Osamah S., Paschalis C. Sofotasios, Sami Muhaidat, Simon L. Cotton, Khaled Rabie, and Naofal Al-Dhahir. "On the secrecy capacity of Fisher-Snedecor F fading channels." In 2018 14th International Conference on Wireless and Mobile Computing, Networking and Communications (WiMob), pp. 102-107. IEEE, 2018.

[16] Ghadi, Farshad Rostami, and Ghosheh Abed Hodtani. "Copula-Based Analysis of Physical Layer Security Performances Over Correlated Rayleigh Fading Channels." IEEE Transactions on Information Forensics and Security 16 (2020): 431-440.

[17] Shannon, Claude E. "Channels with side information at the transmitter." IBM journal of Research and Development 2, no. 4 (1958): 289-293.

[18] Gel'fand, S. I., Pinsker, M. S.,"Coding for Channels with Random Parameters." Probl. Contr. Inform. Theory, 1980, 9, (1), pp. 19-31.

[19] Cover, Thomas M., and Mung Chiang. "Duality between channel capacity and rate distortion with two-sided state information." IEEE Transactions on Information Theory 48, no. 6 (2002): 1629-1638.

[20] Anzabi-Nezhad, Nima S., Ghosheh Abed Hodtani, and Mohammad Molavi Kakhki. "Information theoretic exemplification of the receiver recognition and a more general version for the costa theorem." IEEE Communications letters 17, no. 1 (2012): 107-110.

[21] Anzabi-Nezhad, Nima S., Ghosheh Abed Hodtani, and Mohammad Molavi Kakhki. "A new and more general capacity theorem for the gaussian channel with two-sided input-noise dependent state information." arXiv preprint arXiv:1507.04924 (2015).

[22] Chen, Yanling, and AJ Han Vinck. "Wiretap channel with side information." IEEE Transactions on Information Theory 54, no. 1 (2008): 395-402.

[23] Chia, Yeow-Khiang, and Abbas El Gamal. "Wiretap channel with causal state information." IEEE Transactions on Information Theory 58, no. 5 (2012): 2838-2849.

[24] Liu, Wei, and Biao Chen. "Wiretap channel with two-sided channel state information." In 2007 Conference Record of the Forty-First Asilomar Conference on Signals, Systems and Computers, pp. 893-897. IEEE, 2007.

[25] Xu, Peng, Zhiguo Ding, Xuchu Dai, and Kin K. Leung. "A general framework of wiretap channel with helping interference and state information." IEEE transactions on information forensics and security 9 , no. 2 (2013): 182-195

[26] Gopala, Praveen Kumar, Lifeng Lai, and Hesham El Gamal. "On the secrecy capacity of fading channels." IEEE Transactions on Information Theory 54, no. 10 (2008): 4687-4698.

[27] Aggarwal, Vaneet, Amir Bennatan, and A. Robert Calderbank. "On maximizing coverage in gaussian relay channels." IEEE Transactions on Information Theory 55, no. 6 (2009): 2518-2536.

[28] Alizadeh, Alireza, and Ghosheh Abed Hodtani. "Analysis of capacity and coverage region for Rayleigh fading MIMO relay channel." International Journal of Communication Systems 28, no. 8 (2015): 1462-1474.
[29] Host-Madsen, Anders. "On the capacity of wireless relaying." In Proceedings IEEE 56th Vehicular Technology Conference, vol. 3, pp. 1333-1337. IEEE, 2002.

[30] Jeffrey, Alan, and Daniel Zwillinger, eds. Table of integrals, series, and products. Elsevier, 2007.

[31] Ng, Edward W., and Murray Geller. "A table of integrals of the error functions." Journal of Research of the National Bureau of Standards-B. Mathematical Sciences 73, no. 1 (1969).

[32] Alkheir, Ala Abu, and Mohamed Ibnkahla. "An accurate approximation of the exponential integral function using a sum of exponentials." IEEE communications letters 17, no. 7 (2013): 1364-1367.

[33] Bateman, Harry. "The k-function, a particular case of the confluent hypergeometric function." Transactions of the American Mathematical Society 33, no. 4 (1931): 817-831.

[34] Costa, Max. "Writing on dirty paper (corresp.)." IEEE transactions on information theory 29, no. 3 (1983): 439-441.

[35] Heegard, Chris, and A. El Gamal. "On the capacity of computer memory with defects." IEEE transactions on Information theory 29, no. 5 (1983): 731-739. 